\title{
4d Chern-Simons theory as a 3d Toda theory, and a $3 d-2 d$ correspondence
}

\author{
Meer Ashwinkumar, Kee-Seng Png and Meng-Chwan Tan \\ Department of Physics, National University of Singapore, \\ 2 Science Drive 3, 117551, Singapore \\ E-mail: meerashwinkumar@u.nus.edu, pngkeeseng@u.nus.edu, \\ mctan@nus.edu.sg
}

Abstract: We show that the four-dimensional Chern-Simons theory studied by Costello, Witten and Yamazaki, is, with Nahm pole-type boundary conditions, dual to a boundary theory that is a three-dimensional analogue of Toda theory with a novel $3 \mathrm{~d}$ W-algebra symmetry. By embedding four-dimensional Chern-Simons theory in a partial twist of the five-dimensional maximally supersymmetric Yang-Mills theory on a manifold with corners, we argue that this three-dimensional Toda theory is dual to a two-dimensional topological sigma model with A-branes on the moduli space of solutions to the Bogomolny equations. This furnishes a novel $3 \mathrm{~d}-2 \mathrm{~d}$ correspondence, which, among other mathematical implications, also reveals that modules of the $3 \mathrm{~d} \mathrm{~W}$-algebra are modules for the quantized algebra of certain holomorphic functions on the Bogomolny moduli space.

Keywords: Chern-Simons Theories, Conformal and W Symmetry, Topological Field Theories, Supersymmetric Gauge Theory

ARXIV EPrint: 2008.06053 


\section{Contents}

1 Introduction, summary and conventions 1

2 The starting point: partially twisted 5d MSYM 5

2.1 "GL"-twisted 5d MSYM 5

$\begin{array}{ll}2.2 \text { Boundary conditions } & 7\end{array}$

2.3 Localization to 4d Chern-Simons theory 9

3 3d Toda theory from partially twisted 5d MSYM $\quad 9$

$\begin{array}{ll}3.1 & \text { Derivation of 3d WZW model description of boundary theory }\end{array}$

$\begin{array}{lll}3.2 & \text { Derivation of 3d Toda theory from constrained 3d WZW models } & 13\end{array}$

$\begin{array}{lll}3.3 & \text { Three-dimensional W-algebras } & 15\end{array}$

4 2d twisted sigma model from partially twisted 5d MSYM 19

4.1 2d A-model on Bogomolny moduli space as an effective theory 19

$\begin{array}{ll}4.2 & \text { Physical states of 2d sigma model } \\ \end{array}$

5 The 3d-2d correspondence $\quad 23$

6 Conclusion and future work $\quad 25$

$\begin{array}{ll}\text { A Poisson brackets } & 25\end{array}$

\section{Introduction, summary and conventions}

Introduction. In 2010, the authors in [1] observed a correspondence between two-dimensional Liouville theory and four-dimensional $\mathcal{N}=2$ super Yang-Mills (SYM) with gauge group SU(2). This was termed the AGT correspondence. This correspondence was further investigated for higher-rank gauge groups, where the correspondence is between twodimensional Toda theory and four-dimensional $\mathcal{N}=2 \mathrm{SYM}$ with gauge group $\mathrm{SU}(N)[2]$. Among other approaches, it is known that the AGT correspondence can be understood in terms of the characterization of the space of W-algebra conformal blocks as an irreducible module for quantized algebras of certain holomorphic functions on Hitchin's moduli space [3]. This implies a relationship between Hitchin's moduli space and W-algebra representations.

In this work, we shall present an analogous relationship involving the moduli space of solutions to the Bogomolny equations (which is known to reduce to Hitchin's equations upon dimensional reduction), and a novel $\mathrm{W}$-algebra arising from a three-dimensional analogue of Toda theory. The latter, as we shall show, arises as the boundary dual of the 
four-dimensional Chern-Simons theory studied by Costello, Witten and Yamazaki $[4,5]$ on $I \times S^{1} \times \Sigma$, with Nahm pole-type boundary conditions at the ends of the interval, $I$.

To show the aforementioned relationship, we shall first embed four-dimensional ChernSimons theory in partially-twisted five-dimensional maximally supersymmetric Yang-Mills theory $\left(\mathrm{MSYM}\right.$ ) on $I \times \mathbb{R}_{+} \times S^{1} \times \Sigma$, following [6] (the partial twist being analogous to the geometric Langlands twist of four-dimensional $\mathcal{N}=4 \mathrm{SYM})$. Using the topologicalholomorphic nature of the partially twisted theory, we scale up $I \times \mathbb{R}_{+}$to arrive at a two-dimensional A-twisted supersymmetric sigma model on the moduli space of solutions to the Bogomolny equations defined on $S^{1} \times \Sigma$. This implies a novel $3 \mathrm{~d}-2 \mathrm{~d}$ correspondence, that is, between the sigma model and the aforementioned three-dimensional Toda theory on $S^{1} \times \Sigma$.

Let us now give a brief plan and summary of the paper.

A brief plan and summary of the paper. In section 2, we outline some basic properties of $5 \mathrm{~d}$ MSYM on a manifold of the form $\mathcal{M}=I \times \mathbb{R}_{+} \times S^{1} \times \Sigma$, partially twisted along $I \times \mathbb{R}_{+} \times S^{1}$. We choose a topological twist analogous to the geometric Langlands twist in $4 \mathrm{~d} \mathcal{N}=4$ super Yang-Mills. The action of the $5 \mathrm{~d}$ "GL"-twisted MSYM takes the form

$$
S=\{\mathcal{Q}, \tilde{V}\}+\frac{\widetilde{\Psi}}{4 \pi} \int_{I \times \mathbb{R}_{+} \times S^{1} \times \Sigma} d z \wedge \operatorname{Tr}(\mathcal{F} \wedge \mathcal{F})
$$

In what follows, we shall take the gauge group to be $G=\mathrm{SU}(N)$.

At the origin of $\mathbb{R}_{+}$, we shall choose NS5-type boundary conditions to maintain supersymmetry. At infinity along $\mathbb{R}_{+}$, the boundary conditions are taken to be $\mathcal{Q}$-invariant configurations that are independent of the coordinate parameterizing $\mathbb{R}_{+}$.

At the two ends of the interval, we choose Nahm pole-type boundary conditions of the form

$$
\begin{aligned}
& \mathcal{A} \rightarrow \frac{i d \sigma}{\sigma} H+\frac{d x^{+}}{\sigma} T_{+} \\
& \mathcal{A} \rightarrow \frac{-i d \sigma}{\sigma-\pi} H+\frac{d x^{-}}{\sigma-\pi} T_{-}
\end{aligned}
$$

where $x^{ \pm}$are certain light-cone-like coordinates on $S^{1} \times \Sigma$, and where $\sigma$ parameterizes the interval $I=[0, \pi]$. Here, $H$ and $T_{ \pm}$are the images of Cartan and ladder operators of an $\mathfrak{s u}(2)$ subalgebra of $\mathfrak{s u}(N)$, respectively.

Furthermore, the corresponding path integral localizes to the path integral of the $4 \mathrm{~d}$ Chern-Simons theory with gauge group $\mathrm{SL}(N, \mathbb{C})$, on $I \times S^{1} \times \Sigma$, which has the action

$$
S_{4 \mathrm{~d} C S}[\mathcal{A}]=\frac{1}{2 \pi \hbar} \int_{I \times S^{1} \times \Sigma} d z \wedge \operatorname{Tr}\left(\mathcal{A} \wedge d \mathcal{A}+\frac{2}{3} \mathcal{A} \wedge \mathcal{A} \wedge \mathcal{A}\right)
$$

where $z$ is a complex coordinate on $\Sigma$.

In section 3 , we derive a $3 \mathrm{~d}$ analogue of Toda theory from our partially-twisted $5 \mathrm{~d}$ MSYM. Having localized to 4d Chern-Simons theory on $I \times S^{1} \times \Sigma$, the Nahm pole-type boundary conditions coincide with boundary conditions relating the $4 \mathrm{~d}$ CS theory to a 
constrained 3d WZW model on each boundary. These constrained 3d WZW models are then shown to lead to the $3 \mathrm{~d}$ Toda theory with action

$$
S_{3 \mathrm{~d} \text { Toda }}[\phi]=\frac{1}{2 \pi \hbar} \int_{S^{1} \times \Sigma} d z \wedge d x^{+} \wedge d x^{-}\left(C_{i j} \partial_{+} \phi^{i} \partial_{-} \phi^{j}-4 \sum_{i} \mu_{i} \nu_{i} \mathrm{e}^{C_{i j} \phi_{j}}\right)
$$

Via an equivalent, gauged version of the $3 \mathrm{~d}$ WZW model, we also derive a $3 \mathrm{~d}$ analogue of the Virasoro algebra from 3d Liouville theory, i.e., the $3 \mathrm{~d}$ Toda theory arising from the $4 \mathrm{~d}$ CS theory with SL2, $\mathbb{C}$ gauge group. This is of the form

$$
\begin{aligned}
{\left[\Theta\left(z^{\prime}, \xi^{\prime}\right), \Theta(z, \xi)\right]=} & \left(\partial_{\xi} \Theta(z, \xi) \delta\left(\xi-\xi^{\prime}\right)+2 \Theta(z, \xi) \delta^{\prime}\left(\xi-\xi^{\prime}\right)\right. \\
& \left.+\left(k-\frac{\eta^{2}}{2}\right) \delta^{\prime \prime \prime}\left(\xi-\xi^{\prime}\right)+k \delta^{\prime}\left(\xi-\xi^{\prime}\right)\right) \delta\left(z-z^{\prime}\right)
\end{aligned}
$$

where $\xi=x^{+}$or $x^{-}$. This derivation can be generalized to $\operatorname{SL}(N, \mathbb{C})$ gauge group in a straightforward manner, whereby we would obtain a $3 \mathrm{~d}$ analogue of $\mathrm{W}$-algebras.

In section 4, we derive a topological sigma model with A-branes from the partially twisted 5d MSYM. By exploiting the topological-holomorphic properties of the 5d "GL"twisted theory, we scale up $I \times \mathbb{R}_{+}$. The bosonic part of the $5 \mathrm{~d}$ action then becomes the action of a sigma model of maps $X: I \times \mathbb{R}_{+} \rightarrow \mathcal{M}_{\mathrm{B}}\left(G, S^{1} \times \Sigma\right)$ which takes the form

$$
S_{5 \mathrm{~d} G \mathrm{GL}}^{(A, \varphi)}=\int_{I \times \mathbb{R}_{+}} d^{2} x G_{I J}^{\mathrm{B}} \partial^{\widetilde{\alpha}} X^{I} \partial_{\widetilde{\alpha}} X^{J}
$$

where $\mathcal{M}_{\mathrm{B}}\left(G, S^{1} \times \Sigma\right)$ is the moduli space of solutions to the Bogomolny equations on $S^{1} \times \Sigma$ which take the form

$$
\begin{aligned}
F_{\tau \bar{z}}-i D_{\bar{z}} \varphi_{\tau} & =0 \\
D_{\tau} \varphi^{\tau}-2 i F_{z \bar{z}} & =0
\end{aligned}
$$

where $\tau$ parameterizes $S^{1}$. Furthermore, a term in the non- $\mathcal{Q}$-exact sector of the $5 \mathrm{~d}$ action reduces to an integral over a pullback to $I \times \mathbb{R}_{+}$of a symplectic form on $\mathcal{M}_{\mathrm{B}}\left(G, S^{1} \times \Sigma\right)$, i.e., $\omega=\widetilde{\Psi} \omega_{K}^{B}$, with

$$
\omega_{K}^{\mathrm{B}}=\frac{1}{2 \pi} \int_{S^{1} \times \Sigma} d^{3} x \operatorname{Tr}\left(\delta \phi_{\tau} \wedge \delta A_{4}-\delta A_{5} \wedge \delta A_{\tau}\right)
$$

where $A_{4}=A_{z}+A_{\bar{z}}$ and $A_{5}=i\left(A_{z}-A_{\bar{z}}\right)$. This implies that the sigma model we derived is an A-model depending on the corresponding symplectic structure. We then compute the space of physical states of the A-model to be the space of certain holomorphic sections of bundles defined on Lagrangian branes denoted $\mathcal{B}_{\text {Op }}$ and $\mathcal{B}_{\text {Op }}^{\prime}$, i.e., $H^{0}\left(\mathcal{B}_{\text {Op }}, K_{\mathcal{B}_{\text {Op }}}^{1 / 2}\right) \otimes$ $H^{0}\left(\mathcal{B}_{\text {Op }}^{\prime}, K_{\mathcal{B}_{\mathrm{Op}}^{\prime}}^{1 / 2}\right)$. These physical states are further identified with modules for the quantized algebra of certain holomorphic functions on $\mathcal{M}_{\mathrm{B}}\left(G, S^{1} \times \Sigma\right)$.

In section 5, via the topological-holomorphic property of the parent theory, i.e. partially twisted MSYM on $\mathcal{M}=I \times \mathbb{R}_{+} \times S^{1} \times \Sigma$, we identify 3d Toda states with physical states of the $2 \mathrm{~d}$ A-model with target space $\mathcal{M}_{\mathrm{B}}\left(G, S^{1} \times \Sigma\right)$. Hence, we establish a novel 


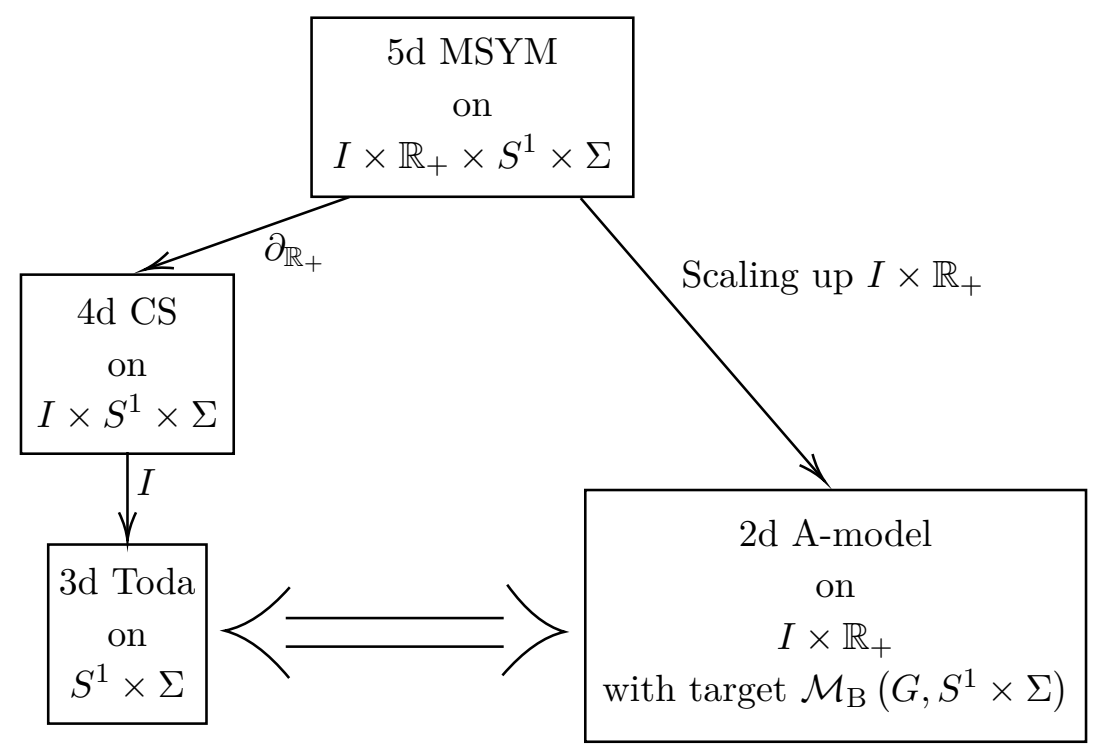

Figure 1. Outline of the steps in this paper. Starting from "GL"-twisted 5d MSYM, we are able to establish a novel correspondence between the $3 \mathrm{~d}$ Toda theory and a $2 \mathrm{~d}$ A-twisted sigma model governing maps $I \times \mathbb{R}_{+} \rightarrow \mathcal{M}_{\mathrm{B}}\left(G, S^{1} \times \Sigma\right)$.

correspondence between the $3 \mathrm{~d}$ Toda theory and the $2 \mathrm{~d}$ A-twisted sigma model with target space $\mathcal{M}_{\mathrm{B}}\left(G, S^{1} \times \Sigma\right)$, i.e., we have

3d Toda theory

on

$$
S^{1} \times \Sigma
$$

Topological sigma model with A-branes

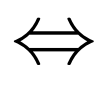

on

$I \times \mathbb{R}_{+}$

with target $\mathcal{M}_{\mathrm{B}}\left(G, S^{1} \times \Sigma\right)$

Mathematically, this implies that

Modules of 3d W-algebras

defined on

$$
\Leftrightarrow \quad H^{0}\left(\mathcal{B}_{\mathrm{Op}}, K_{\mathcal{B}_{\mathrm{Op}}}^{1 / 2}\right) \otimes H^{0}\left(\mathcal{B}_{\mathrm{Op}}^{\prime}, K_{\mathcal{B}_{\mathrm{Op}}^{\prime}}^{1 / 2}\right)
$$

$$
S^{1} \times \Sigma
$$

In other words,

Modules of $3 \mathrm{~d}$ W-algebras defined on $S^{1} \times \Sigma$ are modules for the quantized algebra of certain holomorphic functions on $\mathcal{M}_{\mathrm{B}}\left(G, S^{1} \times \Sigma\right)$.

The steps that we just outlined are summarized in figure 1 .

Labeling conventions. The labeling conventions for the indices used in this paper are as follows: 


\begin{tabular}{c|c}
\hline Number of dimensions & Label \\
\hline $5 \mathrm{~d}\left(\right.$ on $\left.I \times \mathbb{R}_{+} \times S^{1} \times \Sigma\right)$ & $M, N, \cdots=1,2,3,4,5$ \\
\hline 3d $\left(\right.$ on $\left.I \times \mathbb{R}_{+} \times S^{1}\right)$ & $\alpha, \beta, \cdots=1,2,3$ \\
\hline 3d $\left(\right.$ on $\left.S^{1} \times \Sigma\right)$ & $p, q, \cdots=3,4,5$ \\
\hline $2 \mathrm{~d}\left(\right.$ on $\left.I \times \mathbb{R}_{+}\right)$ & $\widetilde{\alpha}, \widetilde{\beta}, \cdots=1,2$ \\
\hline $2 \mathrm{~d}\left(\right.$ on $\left.I \times S^{1}\right)$ & $\dot{\alpha}, \hat{\beta}, \cdots=1,3$ \\
\hline
\end{tabular}

\section{The starting point: partially twisted 5d MSYM}

\section{1 "GL"-twisted 5d MSYM}

The classical action of 5d maximally supersymmetric Yang-Mills (MSYM) theory is of the form $[7,8]$

$$
\begin{aligned}
S= & -\frac{1}{g_{5 \mathrm{~d}}^{2}} \int_{\mathcal{M}} d^{5} x \operatorname{Tr}\left(\frac{1}{4} F_{M N} F^{M N}+\frac{1}{2} D_{M} \varphi_{\widehat{M}} D^{M} \varphi^{\widehat{M}}+\frac{1}{4}\left[\varphi_{\widehat{M}}, \varphi_{\widehat{N}}\right]\left[\varphi^{\widehat{M}}, \varphi^{\widehat{N}}\right]\right. \\
& \left.+i \rho^{\bar{M}} \widehat{\widehat{M}}\left(\Gamma^{M}\right)_{\bar{M}}{ }^{\bar{N}} D_{M} \rho_{\bar{N} \widehat{\bar{M}}}+\rho^{\bar{M} \widehat{\bar{M}}}\left(\Gamma^{\widehat{M}}\right)_{\widehat{\bar{M}}} \widehat{\widehat{\bar{N}}}\left[\varphi_{\widehat{M}}, \rho_{\bar{M} \widehat{\bar{N}}}\right]\right)
\end{aligned}
$$

where $M, N=1, \cdots, 5$. Barred and hatted versions, i.e., $\bar{M}$ and $\widehat{M}$, take the meaning of spinor and R-symmetry indices, respectively. This action is invariant under the supersymmetry transformations

$$
\begin{aligned}
\delta A_{M}= & i \zeta^{\bar{M} \widehat{\bar{M}}}\left(\Gamma_{M}\right)_{\bar{M}}{ }^{\bar{N}} \rho_{\bar{N} \widehat{\bar{M}}} \\
\delta \varphi^{\widehat{M}}= & \zeta^{\bar{M} \widehat{\bar{M}}}\left(\Gamma^{\widehat{M}}\right)_{\widehat{\bar{M}}}{ }^{\widehat{\widehat{N}}} \rho_{\bar{M} \widehat{\bar{N}}} \\
\delta \rho_{\bar{M} \widehat{\bar{M}}}= & -i\left(\Gamma^{M}\right)_{\bar{M}}{ }^{\bar{N}} D_{M} \varphi^{\widehat{M}}\left(\Gamma_{\widehat{M}}\right)_{\widehat{\bar{M}}} \widehat{\bar{N}}_{\bar{N} \widehat{\bar{N}}}-\frac{1}{2}\left(\Gamma_{\widehat{M}}\right)_{\widehat{\bar{M}}} \widehat{\bar{N}}\left(\Gamma_{\widehat{N}}\right)_{\widehat{\bar{N}} \widehat{\bar{L}}}\left[\varphi^{\widehat{M}}, \varphi^{\widehat{N}}\right] \zeta_{\bar{M}} \overline{\bar{L}} \\
& +\frac{1}{2} F^{M N}\left(\Gamma_{M N}\right)_{\bar{M}} \overline{\bar{N}}_{\bar{N} \widehat{\bar{M}}} .
\end{aligned}
$$

We shall take the underlying five-manifold to be of the form $\mathcal{M}=V \times \Sigma$, where submanifolds $V$ and $\Sigma$ correspond to the $\left\{x^{1}, x^{2}, x^{3}\right\}$ and $\left\{x^{4}, x^{5}\right\}$ directions, respectively. In what follows, we shall also take the gauge group to be $G=\mathrm{SU}(N)$. To be consistent with the conventions used in $[6,9]$, we shall also take the Lie algebra $\mathfrak{g}$ to be anti-hermitian, i.e., $U^{\dagger}=-U$ where $U \in \mathfrak{g}$.

We would like to carry out a partial topological twist on the submanifold $V$. For the purpose of deriving the results seen in this paper, we shall choose the twist analogous to the geometric Langlands (GL) twist [9]. The symmetry group of 5d MSYM is $\operatorname{SO}_{\mathcal{M}}(5) \times$ $\mathrm{SO}_{\mathrm{R}}(5)$, where the subscripts denote rotation and R-symmetry groups, respectively.

Prior to carrying out the topological twist, we decompose the rotation group as

$$
\mathrm{SO}_{\mathcal{M}}(5) \rightarrow \mathrm{SO}_{V}(3) \times \mathrm{SO}_{\Sigma}(2),
$$


and the R-symmetry group as

$$
\mathrm{SO}_{\mathrm{R}}(5) \rightarrow \mathrm{SO}_{\mathrm{R}}(3) \times \mathrm{SO}_{\mathrm{R}}(2),
$$

where $\mathrm{SO}_{\mathrm{R}}(3) \subset \mathrm{SO}_{\mathrm{R}}(5)$ rotates the scalar fields $\left\{\varphi_{\widehat{1}}, \varphi_{\widehat{2}}, \varphi_{\widehat{3}}\right\}$.

Carrying out the topological twist described earlier amounts to taking the diagonal subgroup of $\mathrm{SO}_{V}(3)$ and $\mathrm{SO}_{\mathrm{R}}(3)$, i.e., take

$$
\mathrm{SO}_{V}(3)^{\prime} \subset \mathrm{SO}_{V}(3) \times \mathrm{SO}_{\mathrm{R}}(3),
$$

so that the total symmetry group is now $\mathrm{SO}_{V}(3)^{\prime} \times \mathrm{SO}_{\Sigma}(2) \times \mathrm{SO}_{\mathrm{R}}(2)$. The twisting of fermions which transform as $(\mathbf{2}, \mathbf{2})$ under $\mathrm{SO}_{V}(3) \times \mathrm{SO}_{\mathrm{R}}(3)$ means that they now transform as $\mathbf{1} \oplus \mathbf{3}$ under $\mathrm{SO}_{V}(3)^{\prime}$. Moreover, the scalar fields now transform as the components $\left\{\varphi_{1}, \varphi_{2}, \varphi_{3}\right\}$ of a one-form on $V$.

As shown in [6], for an appropriate choice of supercharge, $\mathcal{Q}$, that is scalar along $V$, this theory is the $5 \mathrm{~d}$ analogue of the GL-twisted $4 \mathrm{~d} \mathcal{N}=4$ SYM studied in [9]. ${ }^{1}$ The supercharge can be expressed as

$$
\mathcal{Q}=u \mathcal{Q}_{L}+v \mathcal{Q}_{R}
$$

where $u, v \in \mathbb{C}$, and the labels $L$ and $R$ are reminiscent of the corresponding left- and right- handed supersymmetries in $4 \mathrm{~d}$ GL-twisted theory. By multiplying in a factor of $1 / u$, supersymmetry variations can be rescaled so that the supercharges depend only on the ratio $t=v / u$, i.e., take

$$
\mathcal{Q}_{t}=\mathcal{Q}_{L}+t \mathcal{Q}_{R}
$$

where the ratio $t \in \mathbb{C P}^{1}$ is the analogue of the twisting parameter in the $4 \mathrm{~d}$ GL-twisted theory [9]. In fact, taking $\Sigma=\mathbb{R} \times S^{1}$ or $T^{2}$, whereby the $x^{5}$ direction is $S^{1}$, we can dimensionally reduce along the circle to obtain precisely the transformations used by Kapustin and Witten in [9] via $A_{5} \rightarrow \varphi_{4}$, and similar identifications for the fermionic fields [6]. Moreover, for $t \neq \pm i$, one finds that the $\mathcal{Q}$-transformations only depend on $\Sigma$ via its complex structure. Hence, such a theory is topological on the submanifold $V$, but has holomorphic dependence on the remaining two directions along $\Sigma$.

In what follows, we shall be interested in $V=I \times \mathbb{R}_{+} \times S^{1}$ and $\Sigma=\mathbb{C P}^{1}, \mathbb{C}^{\times}$or $\mathbb{C} /(\mathbb{Z}+\tau \mathbb{Z})$. Such an $\mathcal{M}$ is a manifold with two corners. We shall also pick $t=-1$ for our purposes. The action of the $5 \mathrm{~d}$ "GL"-twisted theory that we study can be written as

$$
S=\{\mathcal{Q}, \widetilde{V}\}+\frac{\widetilde{\Psi}}{4 \pi} \int_{I \times \mathbb{R}_{+} \times S^{1} \times \Sigma} d z \wedge \operatorname{Tr}(\mathcal{F} \wedge \mathcal{F})
$$

where $\widetilde{\Psi}$ is a real parameter, since we have selected $t=-1$. The curvature $\mathcal{F}$ is defined in terms of the complex connection involving $\mathcal{A}_{\alpha}=A_{\alpha}+i \varphi_{\alpha}$ and $\mathcal{A}_{\bar{z}}=A_{\bar{z}}=\frac{1}{2}\left(A_{4}+i A_{5}\right)$, where $z=x^{4}+i x^{5}$ and $\bar{z}=x^{4}-i x^{5}$ are local complex coordinates on $\Sigma$, and where $\alpha=1,2,3$. To arrive at (2.8), one partially twists the physical action (2.1), and finds

\footnotetext{
${ }^{1}$ Such a partial topological twist has also been discussed conceptually by Elliot and Pestun [10].
} 
that it can be expressed in terms of a $\mathcal{Q}$-exact term, $\{\mathcal{Q}, \widetilde{V}\}$, and a term that is not $\mathcal{Q}$ invariant due to the presence of the boundaries. Additional interaction terms ought to be added to arrive at (2.8), where the second term is $\mathcal{Q}$-invariant, as long as $\mathcal{Q}\left(\mathcal{A}_{\alpha}\right)=0$ and $\mathcal{Q}\left(\mathcal{A}_{\bar{z}}\right)=0$ at the boundaries, and the corresponding connection obeys the Bianchi identity. The boundary conditions we choose satisfy these requirements, as we shall explain in the next subsection.

\subsection{Boundary conditions}

Since our five-dimensional gauge theory is defined on a manifold with boundaries, we ought to specify boundary conditions at each of these boundaries.

Firstly, we shall choose an NS5-type boundary condition at the origin of $\mathbb{R}_{+}$. This boundary condition requires that $t$ is real-valued, and includes the conditions that

$$
\begin{aligned}
& \mathcal{Q}\left(\mathcal{A}_{\dot{\alpha}}\right)=0 \\
& \mathcal{Q}\left(\mathcal{A}_{\bar{z}}\right)=0,
\end{aligned}
$$

where $\dot{\alpha}=1,3$, and $\mathcal{A}_{\dot{\alpha}}$ and $\mathcal{A}_{\bar{z}}$ obey Neumann boundary conditions. The fields $A_{2}$ and $\phi_{2}$ instead obey Dirichlet boundary conditions. This NS5-type boundary condition reduces, upon dimensional reduction along $x^{5}$ (for $\Sigma=\mathbb{R} \times S^{1}$ or $T^{2}$ ), to the "topological" NS5-type boundary condition considered in [11], where the choice of $t=-1$ implies that $\Psi$, the $4 \mathrm{~d}$ analogue of $\widetilde{\Psi}$, is real.

Secondly, at infinity along $\mathbb{R}_{+}$, the boundary conditions are taken to be $x^{2}$-independent $\mathcal{Q}$-invariant configurations.

Thirdly, there are two boundaries at the endpoints of $I$, and we shall impose analogous boundary conditions for both of them. To define these boundary conditions we shall define the convenient coordinates

$$
x^{ \pm}=\tau \pm \frac{i}{2} \bar{z},
$$

where $x^{3}=\tau$ parameterizes $S^{1}$. The corresponding partial derivatives and complexified gauge fields are

$$
\begin{aligned}
\partial_{ \pm} & =\frac{1}{2}\left(\partial_{\tau} \mp 2 i \partial_{\bar{z}}\right) \\
\mathcal{A}_{ \pm} & =\frac{1}{2}\left(\mathcal{A}_{\tau} \mp 2 i \mathcal{A}_{\bar{z}}\right),
\end{aligned}
$$

respectively. The boundary conditions at the endpoints of the interval are Nahm pole-type boundary conditions, and can be defined as follows. As we approach one end of the interval, namely $\sigma \rightarrow 0$, where $x^{1}=\sigma \in I=[0, \pi]$, we impose boundary conditions that include

$$
\mathcal{A} \rightarrow \frac{i d \sigma}{\sigma} H+\frac{d x^{+}}{\sigma} T_{+}
$$


Here, for $\mathfrak{s u}(2) \subset \mathfrak{s u}(n)$, a homomorphism $\rho: \mathfrak{s u}(2) \rightarrow \mathfrak{s u}(N)$ has been chosen, such that the image, $T_{+}$, of the raising operator of $\mathfrak{s u}(2)$ is a maximal length Jordan block, i.e.,

$$
T_{+}=-i\left(\begin{array}{ccccc}
0 & \mu_{1} & 0 & \cdots & 0 \\
0 & 0 & \mu_{2} & \cdots & 0 \\
\vdots & \vdots & \vdots & \ddots & \vdots \\
0 & 0 & 0 & \cdots & \mu_{N-1} \\
0 & 0 & 0 & \cdots & 0
\end{array}\right)
$$

where the imaginary number $i$ accounts for the anti-hermiticity of fields in the 5d "GL"twisted theory, and where $\mu_{i}$ are real and non-zero constants. Imposing the boundary condition (2.13) implies that

$$
\mathcal{A}_{-}=0
$$

at $\sigma=0$.

On the other end of the interval, where $\sigma \rightarrow \pi$, we impose boundary conditions that include

$$
\mathcal{A} \rightarrow \frac{-i d \sigma}{\sigma-\pi} H+\frac{d x^{-}}{\sigma-\pi} T_{-}
$$

Here, for $\mathfrak{s u}(2) \subset \mathfrak{s u}(n)$ we choose a homomorphism $\rho^{\prime}: \mathfrak{s u}(2) \rightarrow \mathfrak{s u}(N)$ such that the image, $T_{-}$, of the lowering operator is a maximal length Jordan block, i.e.,

$$
T_{-}=-i\left(\begin{array}{ccccc}
0 & 0 & \cdots & 0 & 0 \\
\nu_{1} & 0 & \cdots & 0 & 0 \\
0 & \nu_{2} & \cdots & 0 & 0 \\
\vdots & \vdots & \ddots & \vdots & \vdots \\
0 & 0 & \cdots & \nu_{N-1} & 0
\end{array}\right),
$$

where $\nu_{i}$ are real and non-zero constants. The boundary condition (2.16) implies that we set

$$
\mathcal{A}_{+}=0
$$

at $\sigma=\pi$.

In order for these Nahm pole-type boundary conditions at the two ends of the interval to be well-defined, we also require that $\mathcal{A}_{\sigma}, \mathcal{A}_{\tau}$, and $\mathcal{A}_{\bar{z}}$ are $\mathcal{Q}$-invariant at these boundaries. It is crucial that all our boundary conditions are consistent at the corners of our five-manifold, and the fact that $\mathcal{A}_{\alpha}$ and $\mathcal{A}_{\bar{z}}$ are $\mathcal{Q}$-invariant (or zero) at all the boundaries ensures this.

It can be easily shown that upon dimensional reduction on $S^{1} \subset \Sigma$ (for $\Sigma=\mathbb{R} \times S^{1}$ or $T^{2}$ ), the boundary conditions (2.13) and (2.16) reduce to

$$
\mathcal{A} \rightarrow \frac{i d \sigma}{\sigma} H+\frac{d u}{\sigma} T_{+}
$$

and

$$
\mathcal{A} \rightarrow \frac{-i d \sigma}{\sigma-\pi} H+\frac{d \bar{u}}{\sigma-\pi} T_{-}
$$


respectively, where $u=\tau+i x^{4}$ and $\bar{u}=\tau-i x^{4}$ are complex coordinates. These are known, from [12], to be complex Nahm pole boundary conditions for the 4d GL-twisted theory. Moreover, the resulting corner configurations at the intersection of the origin of $\mathbb{R}_{+}$and the endpoints of the interval have been argued to be consistent in [11].

Having specified all our boundary conditions, we can observe that the $\mathcal{Q}$-invariance of the second term in (2.8) follows from the fact that $\mathcal{A}_{\alpha}$ and $\mathcal{A}_{\bar{z}}$ are $\mathcal{Q}$-invariant (or zero) at all the boundaries and the fact that the Bianchi identity is valid everywhere on the five-manifold. The latter is true since the Nahm pole-like configurations are complex flat connections, and therefore there are no singularities in their curvatures.

\subsection{Localization to $4 \mathrm{~d}$ Chern-Simons theory}

The path integral of the $5 \mathrm{~d}$ partially-twisted theory localizes to solutions of $\mathcal{Q}$-invariant configurations, whereby it reduces to a path integral over the non- $\mathcal{Q}$-exact term in (2.8). Via Stokes' theorem, and the boundary conditions at each boundary, one finds that this term becomes a nontrivial $\mathcal{Q}$-invariant boundary term at the origin of $\mathbb{R}_{+}$(that takes the form of the $4 \mathrm{~d}$ Chern-Simons action), together with a constant term at infinity along $\mathbb{R}_{+}$. The boundary conditions at the endpoints of $I$ set the $4 \mathrm{~d}$ Chern-Simons actions there to zero. As a result, up to normalization, one arrives at the path integral of $4 \mathrm{~d}$ Chern-Simons (CS) theory, which takes the form

$$
\int_{\widetilde{\Gamma}} D \mathcal{A} \exp \left(\frac{i}{2 \pi \hbar} \int_{I \times S^{1} \times \Sigma} d z \wedge \operatorname{Tr}\left(\mathcal{A} \wedge d \mathcal{A}+\frac{2}{3} \mathcal{A} \wedge \mathcal{A} \wedge \mathcal{A}\right)\right),
$$

where $\widetilde{\Gamma}$ is the integration cycle defined by the $\mathcal{Q}$-invariant localization equations, and $\frac{i}{\hbar}=\frac{\widetilde{\Psi}}{2}$. This integration cycle is a Lefschetz thimble that ensures the convergence of the path integral. Here, the gauge connection is defined by $\mathcal{A}=\mathcal{A}_{\sigma} d \sigma+\mathcal{A}_{\tau} d \tau+\mathcal{A}_{\bar{z}} d \bar{z}$.

Hence, the ensuing theory on the boundary of $\mathbb{R}_{+}$is the $4 \mathrm{~d}$ Chern-Simons theory, which has the action

$$
S_{4 \mathrm{~d} C S}[\mathcal{A}]=\frac{1}{2 \pi \hbar} \int_{I \times S^{1} \times \Sigma} d z \wedge \operatorname{Tr}\left(\mathcal{A} \wedge d \mathcal{A}+\frac{2}{3} \mathcal{A} \wedge \mathcal{A} \wedge \mathcal{A}\right)
$$

\section{3d Toda theory from partially twisted 5d MSYM}

Consider the 4d CS theory on a four-manifold with a single boundary along the topological plane. If one of the complex gauge fields (namely $\mathcal{A}_{\bar{z}}$ ) is set to vanish at the boundary, a 3d analogue of the chiral Wess-Zumino-Witten (WZW) model can be obtained [13].

In the $4 \mathrm{~d}$ CS theory defined in (2.22), the underlying manifold has two boundaries at the ends of $I$, where we have Nahm pole-type boundary conditions that, among other things, also constrain some gauge fields to vanish, i.e., $\mathcal{A}_{-}=0$ and $\mathcal{A}_{+}=0$ at $\sigma=0$ and $\sigma=\pi$, respectively (see (2.15) and (2.18)). Thus, we expect that the $4 \mathrm{~d}$ CS theory in our present analysis can be described by 3d WZW models at the boundaries, with further constraints coming from the Nahm pole-type boundary conditions.

In what follows, we shall first derive $3 \mathrm{~d}$ WZW models defined at the boundaries of $I$, and then include the further constraints that originate from the Nahm pole-type boundary conditions to obtain a $3 \mathrm{~d}$ analogue of analytically-continued Toda theory. 


\subsection{Derivation of 3d WZW model description of boundary theory}

3d WZW Model at $\boldsymbol{\sigma} \rightarrow \mathbf{0}$. We shall first verify the locality and gauge invariance of the $4 \mathrm{~d}$ CS theory defined in (2.22), near the boundary at $\sigma=0$. To show the locality of our $4 \mathrm{~d}$ CS theory here, we first vary the action (2.22) to give

$$
\delta S_{4 \mathrm{~d} C S}=\frac{1}{2 \pi \hbar} \int_{I \times S^{1} \times \Sigma} d z \wedge \operatorname{Tr}(\delta \mathcal{A} \wedge \mathcal{F}+d(\delta \mathcal{A} \wedge \mathcal{A})) .
$$

Using Stokes' theorem, the second term of the variation (3.1) is identified as a boundary term. Imposing the boundary condition $\mathcal{A}_{-}=0$ (see (2.15)), the boundary variation vanishes.

To show the gauge invariance of our 4d CS theory, we first extend the partial connection $\mathcal{A}$ to a full connection, which allows us to rewrite (2.22) as

$$
S_{4 \mathrm{~d} \mathrm{CS}}[\mathcal{A}]=-\frac{1}{2 \pi \hbar} \int_{I \times S^{1} \times \Sigma} z \operatorname{Tr}(\mathcal{F} \wedge \mathcal{F})+\frac{1}{2 \pi \hbar} \int_{S^{1} \times \Sigma} z \operatorname{Tr}\left(\mathcal{A} \wedge d \mathcal{A}+\frac{2}{3} \mathcal{A} \wedge \mathcal{A} \wedge \mathcal{A}\right)
$$

Using the boundary condition $\mathcal{A}_{-}=0$, and imposing the additional boundary condition $\mathcal{A}_{z}=0$, the second term of (3.2) vanishes (it should be noted that we are not taking $\mathcal{A}_{z}$ to be the complex conjugate of $\mathcal{A}_{\bar{z}}$ ). We then find that the first term is gauge-invariant under large gauge transformations of the form

$$
\mathcal{A} \rightarrow U \mathcal{A} U^{-1}-d U U^{-1}
$$

However, we ought to restrict $U$ such that the boundary conditions $\mathcal{A}_{-}=0=\mathcal{A}_{z}$ are preserved. We shall achieve this by taking $U$ to tend to the identity element of $G$ at $\sigma=0$.

Now, in order to derive the 3d WZW model at the boundary at $\sigma=0$, we exploit the fact that the $4 \mathrm{~d}$ CS action (2.22) admits an alternative form

$$
\frac{1}{2 \pi \hbar} \int_{I \times S^{1} \times \Sigma} d z \wedge d \sigma \wedge d x^{-} \wedge d x^{+} \operatorname{Tr}\left(2 \mathcal{A}_{-} \mathcal{F}_{+\sigma}-\mathcal{A}_{\sigma} \partial_{-} \mathcal{A}_{+}+\mathcal{A}_{+} \partial_{-} \mathcal{A}_{\sigma}\right),
$$

as $\sigma \rightarrow 0$, where it can be seen that $\mathcal{A}_{-}$is a Lagrange multiplier. In turn, the Lagrange multiplier is then integrated out to produce the condition $\mathcal{F}_{+\sigma}=0$, which has solutions that can be expressed as

$$
\begin{aligned}
& \mathcal{A}_{\sigma}=g^{-1} \partial_{\sigma} g \\
& \mathcal{A}_{+}=g^{-1} \partial_{+} g,
\end{aligned}
$$

where $g$ is a map, $g: I \times S^{1} \times \Sigma \rightarrow S L(N, \mathbb{C})$. Changing variables from $\mathcal{A}_{\sigma}$ and $\mathcal{A}_{+}$to $g,(2.22)$ then becomes

$$
\begin{aligned}
\lim _{\sigma \rightarrow 0} S_{4 \mathrm{~d} \mathrm{CS}}[\mathcal{A}]= & S_{3 \mathrm{~d} \text { WZW }}[g] \\
= & \frac{1}{2 \pi \hbar} \int_{S^{1} \times \Sigma} d z \wedge d x^{+} \wedge d x^{-} \operatorname{Tr}\left(\partial_{+} g g^{-1} \partial_{-} g g^{-1}\right) \\
& -\frac{1}{6 \pi \hbar} \int_{I \times S^{1} \times \Sigma} d z \wedge \operatorname{Tr}\left(d g g^{-1} \wedge d g g^{-1} \wedge d g g^{-1}\right),
\end{aligned}
$$

which takes the form of a $3 \mathrm{~d}$ analogue of the $2 \mathrm{~d}$ chiral WZW model. 
Next, we note that a gauge transformation (3.3) amounts to $g \rightarrow g U^{-1}$ in (3.5). As a result, we may change the value of $g$ in the bulk without changing its value at $\sigma=0$. This means that the Wess-Zumino term in (3.6) does not depend on the choice of extension of the boundary value of $g$ over the bulk manifold. Thus, we can divide out the volume of the gauge group to obtain a path integral of the form

$$
\int D \widetilde{g} \mathrm{e}^{i S[\widetilde{g}]}
$$

where $\widetilde{g}$ is now the map $\widetilde{g}: S^{1} \times \Sigma \rightarrow \mathrm{SL}(N, \mathbb{C})$.

Varying the action (3.6), we obtain

$$
\delta S_{3 \mathrm{~d} \text { WZW }}=-\frac{1}{\pi \hbar} \int_{S^{1} \times \Sigma} d z \wedge d x^{+} \wedge d x^{-} \operatorname{Tr}\left(\widetilde{g}^{-1} \delta \widetilde{g} \partial_{-}\left(\widetilde{g}^{-1} \partial_{+} \widetilde{g}\right)\right),
$$

which implies the equation of motion

$$
\partial_{-}\left(\widetilde{g}^{-1} \partial_{+} \widetilde{g}\right)=0 .
$$

This equation of motion is equivalent to

$$
\partial_{+}\left(\partial_{-} \widetilde{g} \widetilde{g}^{-1}\right)=0
$$

Hence, the solutions of (3.9) and (3.10) take the form

$$
\widetilde{g}\left(z, x^{+}, x^{-}\right)=A\left(z, x^{+}\right) B\left(z, x^{-}\right) .
$$

The equations of motion, (3.9) and (3.10), are the current conservation equations for the symmetry of the action under the transformation

$$
\widetilde{g}\left(z, x^{+}, x^{-}\right) \rightarrow \widetilde{\Omega}\left(z, x^{+}\right) \widetilde{g} \Omega\left(z, x^{-}\right),
$$

where the conserved currents take the form

$$
\begin{aligned}
& J_{+}=\widetilde{g}^{-1} \partial_{+} \widetilde{g} \\
& J_{-}=\partial_{-} \widetilde{g} \widetilde{g}^{-1} .
\end{aligned}
$$

Crucially, it is seen that we can identify $\mathcal{A}_{+}$with $J_{+}$on the boundary at $\sigma=0$, and so, the rest of the boundary conditions in (2.13) also constrain $J_{+}$.

3d WZW Model at $\boldsymbol{\sigma} \rightarrow \boldsymbol{\pi}$. We shall now verify the locality and gauge invariance of the $4 \mathrm{~d}$ CS theory defined in (2.22), near the boundary at $\sigma=\pi$. To show the locality of our $4 \mathrm{~d}$ CS theory here, we first vary the action (2.22). Then, we impose the boundary condition $\mathcal{A}_{+}=0$ (see (2.18)). In doing so, the boundary contribution to the variation (3.1) vanishes.

To show the gauge invariance of our 4d CS theory, we first extend the partial connection $\mathcal{A}$ to a full connection. Using the boundary condition $\mathcal{A}_{+}=0$, and imposing the additional boundary condition $\mathcal{A}_{z}=0$, the second term of (3.2) vanishes. Like before, we ought to 
restrict $U$ such that the boundary conditions $\mathcal{A}_{+}=0=\mathcal{A}_{z}$ are preserved. We shall achieve this by taking $U$ to tend to the identity element of $G$ at $\sigma=\pi$.

Now, in order to derive the 3d WZW model at the boundary at $\sigma=\pi$, we make use of the fact that the $4 \mathrm{~d}$ CS action (2.22) admits an alternative form

$$
\frac{1}{2 \pi \hbar} \int_{I \times S^{1} \times \Sigma} d z \wedge d \sigma \wedge d x^{-} \wedge d x^{+} \operatorname{Tr}\left(2 \mathcal{A}_{+} \mathcal{F}_{-\sigma}+\mathcal{A}_{\sigma} \partial_{+} \mathcal{A}_{-}-\mathcal{A}_{-} \partial_{+} \mathcal{A}_{\sigma}\right)
$$

as $\sigma \rightarrow \pi$. It can be seen that $\mathcal{A}_{+}$is a Lagrange multiplier, which is then integrated out to produce the condition $\mathcal{F}_{\sigma-}=0$. We shall choose solutions to this condition that take the form

$$
\begin{aligned}
& \mathcal{A}_{\sigma}=-\partial_{\sigma} g g^{-1} \\
& \mathcal{A}_{-}=-\partial_{-} g g^{-1},
\end{aligned}
$$

where $g$ is a map, $g: I \times S^{1} \times \Sigma \rightarrow S L(N, \mathbb{C})$.

Changing variables from $\mathcal{A}_{\sigma}$ and $\mathcal{A}_{-}$to $g$, and taking note of the orientation of $I,(2.22)$ then becomes

$$
\begin{aligned}
\lim _{\sigma \rightarrow \pi} S_{4 \mathrm{~d} \mathrm{CS}}[\mathcal{A}]= & S_{3 \mathrm{~d} \text { WZW }}^{\prime}[g] \\
= & \frac{1}{2 \pi \hbar} \int_{S^{1} \times \Sigma} d z \wedge d x^{+} \wedge d x^{-} \operatorname{Tr}\left(\partial_{+} g g^{-1} \partial_{-} g g^{-1}\right) \\
& +\frac{1}{6 \pi \hbar} \int_{I \times S^{1} \times \Sigma} d z \wedge \operatorname{Tr}\left(d g g^{-1} \wedge d g g^{-1} \wedge d g g^{-1}\right),
\end{aligned}
$$

which differs from (3.6) by a minus sign on the topological term.

Next, we note that a gauge transformation (3.3) amounts to $g \rightarrow U g$ in (3.15a). As a result, we may change the value of $g$ in the bulk without changing its value at $\sigma=\pi$. This means that the Wess-Zumino term in (3.16) does not depend on the choice of extension of the boundary value of $g$ over the bulk manifold. Thus, we can divide out the volume of the gauge group to obtain a path integral of the form

$$
\int D \widetilde{g} \mathrm{e}^{i S[\widetilde{g}]}
$$

where $\widetilde{g}$ is now the map $\widetilde{g}: S^{1} \times \Sigma \rightarrow \mathrm{SL}(N, \mathbb{C})$.

Varying the action (3.16), and taking note of the orientation of $I$, we obtain

$$
\delta S_{3 \mathrm{~d} W Z \mathrm{~W}}^{\prime}=-\frac{1}{\pi \hbar} \int_{S^{1} \times \Sigma} d z \wedge d x^{+} \wedge d x^{-} \operatorname{Tr}\left(\widetilde{g}^{-1} \delta \widetilde{g} \partial_{-}\left(\widetilde{g}^{-1} \partial_{+} \widetilde{g}\right)\right),
$$

which is the same as (3.8). This means that identical equations of motion, (3.9) and (3.10), are obtained, and the corresponding conserved currents are proportional to those in (3.13).

With a convenient overall factor, the conserved currents are

$$
\begin{aligned}
& J_{+}^{\prime}=-\widetilde{g}^{-1} \partial_{+} \widetilde{g} \\
& J_{-}^{\prime}=-\partial_{-} \widetilde{g} \widetilde{g}^{-1},
\end{aligned}
$$

where the prime on $J^{\prime}$ reminds the reader that these currents are localized to $\sigma \rightarrow \pi$. Since we can identify $\mathcal{A}_{-}$with $J_{-}^{\prime}$ on the boundary at $\sigma=\pi$, the rest of the boundary conditions in (2.16) also constrain $J_{-}^{\prime}$. 


\subsection{Derivation of 3d Toda theory from constrained 3d WZW models}

Current constraints. Note that $T_{+}$in (2.14) $\left(T_{-}\right.$in (2.17)) can be expressed as a sum of the positive (negative) simple roots with non-zero coefficients. In addition, a current, $J$, can be projected onto Cartan directions, as well as roots of the Lie algebra, i.e., we can write

$$
J=\sum_{\alpha \in \Delta}\left(J^{-\alpha} R_{-\alpha}+J^{+\alpha} R_{+\alpha}\right)+J^{0 i} R_{0 i},
$$

where $R_{0 i}$ denotes the $i$-th Cartan generator for $i=1, \cdots, N-1$, while $\Delta$ denotes the root space. Since $\mu_{i}$ and $\nu_{i}$ are non-zero only for simple roots, $R_{ \pm i}$, the Nahm pole-type boundary conditions (2.13) and (2.16) can be shown to imply that

$$
\begin{aligned}
{J_{+}}^{+i} & =\mu_{i} \\
{J_{+}}^{0 i} & =0
\end{aligned}
$$

and

$$
\begin{aligned}
{J_{-}^{\prime}}^{-i} & =\nu_{i} \\
{J_{-}^{\prime}}^{0 i} & =0,
\end{aligned}
$$

respectively. Together, (3.21) and (3.22) are current constraints. In this sense, what we really derived earlier is a constrained $3 \mathrm{~d}$ WZW model.

Gauss decomposition. Using the Gauss decomposition, the field $\widetilde{g}$ can be expressed as

$$
\widetilde{g}=\exp \left(i \sum_{\alpha \in \Delta} X_{\alpha} R_{\alpha}^{+}\right) \exp \left(i \phi_{i} R_{i}^{0}\right) \exp \left(i \sum_{\alpha \in \Delta} Y_{\alpha} R_{\alpha}^{-}\right),
$$

where $X_{\alpha}, Y_{\alpha}$ and $\phi_{i}$ are scalar fields, and summations are carried out over repeated indices. Using (3.23), $J_{+}=\widetilde{g}^{-1} \partial_{+} \widetilde{g}$ may then be rewritten as

$$
\begin{aligned}
J_{+}= & \sum_{i}\left(i \partial_{+} X_{i} \mathrm{e}^{-C_{i j} \phi_{j}}\right) R_{i}^{+}+\left(-\sum_{i, k} C_{i k} Y_{i} Y_{k} \partial_{+} X_{k} \mathrm{e}^{-C_{k j} \phi_{j}}-i \sum_{i, j} C_{i j} Y_{i} \partial_{+} \phi_{j}+i \sum_{i} \partial_{+} Y_{i}\right) R_{i}^{-} \\
& +\sum_{i}\left(i \partial_{+} \phi_{i}+2 i Y_{i} \partial_{+} X_{i} \mathrm{e}^{-C_{i j} \phi_{j}}\right) R_{i}^{0}+\cdots
\end{aligned}
$$

where $C_{i j}$ are elements of the Cartan matrix, $\exp \left(C_{i j} \phi_{j}\right)=\exp \left(\sum_{j} C_{i j} \phi_{j}\right)$, and where $\cdots$ are terms involving non-simple roots. For $\operatorname{SL}(N, \mathbb{C})$, the Cartan matrix is written as

$$
\left[C_{i j}\right]=\left(\begin{array}{ccccc}
2 & -1 & \cdots & 0 & 0 \\
-1 & 2 & \cdots & 0 & 0 \\
\vdots & \vdots & \ddots & \vdots & \vdots \\
0 & 0 & \cdots & 2 & -1 \\
0 & 0 & \cdots & -1 & 2
\end{array}\right)
$$


Comparing (3.24) with (3.21), we obtain

$$
\begin{aligned}
\partial_{+} X_{i} \mathrm{e}^{-C_{i j} \phi_{j}} & =-i \mu_{i} \\
\partial_{+} \phi_{i}+2 Y_{i} \partial_{+} X_{i} \mathrm{e}^{-C_{i j} \phi_{j}} & =0 .
\end{aligned}
$$

Likewise, $J_{-}^{\prime}=-\partial_{-} \widetilde{g} \widetilde{g}^{-1}$ may be rewritten as

$$
\begin{aligned}
J_{-}^{\prime}= & \left(\sum_{i, k} C_{i k} X_{i} X_{k} \partial_{-} Y_{k} \mathrm{e}^{-C_{k j} \phi_{j}}+i \sum_{i, j} C_{i j} X_{i} \partial_{-} \phi_{j}-i \sum_{i} \partial_{-} X_{i}\right) R_{i}^{+}+\sum_{i}\left(-i \partial_{-} Y_{i} \mathrm{e}^{-C_{i j} \phi_{j}}\right) R_{i}^{-} \\
& +\sum_{i}\left(-i \partial_{-} \phi_{i}-2 i X_{i} \partial_{-} Y_{i} \mathrm{e}^{-C_{i j} \phi_{j}}\right) R_{i}^{0}+\cdots
\end{aligned}
$$

where $\cdots$ are terms involving non-simple roots. Comparing (3.27) with (3.22), we obtain

$$
\begin{aligned}
\partial_{-} Y_{i} \mathrm{e}^{-C_{i j} \phi_{j}} & =i \nu_{i} \\
\partial_{-} \phi_{i}+2 X_{i} \partial_{-} Y_{i} \mathrm{e}^{-C_{i j} \phi_{j}} & =0 .
\end{aligned}
$$

Combining (3.26) and (3.28) then gives

$$
\partial_{+} \partial_{-} \phi_{i}+2 \mu_{i} \nu_{i} \mathrm{e}^{C_{i j} \phi_{j}}=0
$$

which resemble the equations of motion for $2 \mathrm{~d}$ Toda theory. The corresponding action that gives these constraints as equations of motion is

$$
S_{3 \mathrm{~d} \text { Toda }}[\phi]=\frac{1}{2 \pi \hbar} \int_{S^{1} \times \Sigma} d z \wedge d x^{+} \wedge d x^{-}\left(C_{i j} \partial_{+} \phi^{i} \partial_{-} \phi^{j}-4 \sum_{i} \mu_{i} \nu_{i} \mathrm{e}^{C_{i j} \phi_{j}}\right)
$$

which takes the form of a $3 \mathrm{~d}$ analogue of analytically-continued $2 \mathrm{~d}$ Toda theory, and is expected to be an integrable field theory since (3.29) resembles the equations of motion of $2 \mathrm{~d}$ Toda theory, which can be solved exactly. Hence, the edge modes of the $4 \mathrm{~d}$ CS theory with Nahm-pole-type boundary conditions are $3 \mathrm{~d}$ Toda fields.

One may also obtain $3 \mathrm{~d}$ Toda theory from 4d CS theory via an off-shell procedure. The two constrained 3d 'chiral' WZW models can be identified with a single 3d WZW model due to the topological invariance along $I$, and the Gauss decomposition (3.23) can then be substituted into it. Subsequently implementing the constraints (3.26) and (3.28) in the resulting action furnishes the $3 \mathrm{~d}$ Toda action (3.30).

It is expected that if one includes Wilson lines along $I$ or 't Hooft operators along $I \times \mathbb{R}_{+}$in the $5 \mathrm{~d}$ "GL"-twisted theory, one should obtain Wilson and 't Hooft lines along $I$ in the $4 \mathrm{~d}$ Chern-Simons theory that results from localization. These in turn ought to correspond to local operators in the dual $3 \mathrm{~d}$ Toda theory.

Dimensional reduction. If we take $\Sigma=\mathbb{R} \times S^{1}$ or $T^{2}$, one can carry out a dimensional reduction on the circle in the $x^{5}$ direction. The dimensional reduction amounts to setting $\partial_{\bar{z}} \rightarrow \frac{1}{2} \partial_{4}$ and $d z \wedge d \bar{z}=-2 i d x^{4} \wedge d x^{5}$, which implies that $\partial_{+} \rightarrow \partial_{u}=\frac{1}{2}\left(\partial_{\tau}-i \partial_{4}\right)$ and $\partial_{-} \rightarrow \partial_{\bar{u}}=\frac{1}{2}\left(\partial_{\tau}+i \partial_{4}\right)$. The $3 \mathrm{~d}$ Toda action (3.30) then becomes

$$
S_{2 \mathrm{~d} \text { Toda }}[\phi]=\frac{i R}{2 \hbar} \int_{C} d u \wedge d \bar{u}\left(C_{i j} \partial_{u} \phi^{i} \partial_{\bar{u}} \phi^{j}-4 \sum_{i} \mu_{i} \nu_{i} \mathrm{e}^{C_{i j} \phi_{j}}\right),
$$


where we have denoted the remaining two directions by $C$, and $R$ is the radius of the shrunken circle. The action (3.31) indeed takes the form of the usual analytically-continued $2 \mathrm{~d}$ conformal Toda action, as expected.

\subsection{Three-dimensional W-algebras}

In this section, we shall explain how the $3 \mathrm{~d}$ Toda theory gives rise to analogues of $\mathrm{W}$ algebras one usually obtains from $2 \mathrm{~d}$ Toda theory. To this end, we shall first derive the $3 \mathrm{~d}$ analogue of the $2 \mathrm{~d}$ improved energy-momentum tensor, which gives rise to the Virasoro algebra in $2 \mathrm{~d}$ Toda theory.

A 3d analogue of improved energy-momentum tensor. Unlike $2 \mathrm{~d}$ Toda theory, the $3 \mathrm{~d}$ Toda action (3.30) is not invariant under coordinate transformations of the form $x^{p} \rightarrow f\left(x^{p}\right)$, if we assume that $f\left(x^{p}\right)$ are all non-trivial transformations. This is due to the volume element, which transforms as $d^{3} x \rightarrow\left(\partial_{+} f\right)\left(\partial_{-} f\right)\left(\partial_{z} f\right) d^{3} x$, while the $3 \mathrm{~d}$ Toda Lagrangian transforms as $\mathcal{L}_{3 \mathrm{~d} \text { Toda }} \rightarrow \frac{1}{\left(\partial_{+} f\right)\left(\partial_{-} f\right)} \mathcal{L}_{3 \mathrm{~d}}$ Toda. Instead, we shall take only $f\left(x^{+}\right)$ to be a non-trivial transformation, ${ }^{2}$ i.e., take non-trivial transformations only along the $x^{+}$-direction. ${ }^{3}$

Let us now consider infinitesimal coordinate transformations $f\left(x^{+}\right)=x^{+}+\varepsilon\left(x^{+}\right)$where $\varepsilon\left(x^{+}\right) \ll 1$. Since the transformation law for scalars is

$$
\phi_{i}\left(z, x^{+}, x^{-}\right)=\phi_{i}^{\prime}\left(z, x^{\prime+}, x^{-}\right)=\phi_{i}^{\prime}\left(z, x^{+}+\varepsilon\left(x^{+}\right), x^{-}\right)
$$

one would naively look at the corresponding scalar field transformation

$$
\delta \phi_{i}\left(z, x^{+}, x^{-}\right)=\phi_{i}^{\prime}\left(z, x^{+}, x^{-}\right)-\phi_{i}\left(z, x^{+}, x^{-}\right)=-\varepsilon \partial_{+} \phi_{i}\left(z, x^{+}, x^{-}\right) .
$$

However, while the kinetic term in (3.30) remains invariant under this transformation, the potential term does not.

Instead, we shall consider the following modified transformation law for the $3 \mathrm{~d}$ Toda fields

$$
\begin{aligned}
\phi_{i}^{\prime}\left(z, x^{\prime+}, x^{-}\right) & =\phi_{i}\left(z, x^{+}, x^{-}\right)+\left(\sum_{j} C_{i j}^{-1}\right) \ln \partial_{+} f \\
& =\phi_{i}\left(z, x^{+}, x^{-}\right)+\gamma_{i} \ln \partial_{+} f
\end{aligned}
$$

where we have written $\sum_{j} C_{i j}^{-1}=\gamma_{i}$. It can be shown that the $3 \mathrm{~d}$ Toda action remains invariant under the corresponding infinitesimal variations

$$
\phi_{i}\left(z, x^{+}, x^{-}\right) \rightarrow \phi_{i}\left(z, x^{+}, x^{-}\right)-\varepsilon \partial_{+} \phi_{i}+\gamma_{i} \partial_{+} \varepsilon,
$$

whereby the Lagrangian varies as a boundary term.

\footnotetext{
${ }^{2}$ In the following analysis, we shall take $x^{+}$and $x^{-}$to be independent coordinates for simplicity. In doing so, we extend the range of the coordinate $\tau$ such that it is complex-valued. The physical space of interest, however, is defined by $\bar{\tau}=\tau$.

${ }^{3}$ It should also be noted that the choice $f=f\left(x^{-}\right)$is equally valid. In fact, this alternate choice will lead to another copy of the $\mathrm{W}$-algebras that we will derive from the $3 \mathrm{~d}$ Toda theory.
} 
The infinitesimal symmetry variation of the action (3.30) is given explicitly by

$$
\begin{aligned}
\delta S_{3 \mathrm{~d} \text { Toda }}= & \int_{S^{1} \times \Sigma} d z \wedge d x^{+} \wedge d x^{-}\left[\partial_{+}\left(-\frac{1}{2 \pi \hbar}\left(C_{i j} \varepsilon \partial_{+} \phi^{i} \partial_{-} \phi^{j}-2 \sum_{i} \varepsilon \partial_{+} \partial_{-} \phi_{i}\right)\right)\right. \\
& \left.-\partial_{-}\left(\frac{1}{2 \pi \hbar} C_{i j} \gamma^{i} \partial_{+} \partial_{+} \varepsilon \phi^{j}\right)\right] .
\end{aligned}
$$

This is equivalent to the on-shell variation of the action, which is written as

$$
\delta^{\prime} S_{3 \mathrm{~d} \text { Toda }}=\int_{S^{1} \times \Sigma} d z \wedge d x^{+} \wedge d x^{-} \partial_{p}\left(\frac{\partial \mathcal{L}_{3 \mathrm{~d} \text { Toda }}}{\partial\left(\partial_{p} \phi_{i}\right)} \delta \phi_{i}\right) .
$$

We can then derive the Noether current that corresponds to the symmetry of the action under the variation described in (3.35), by equating the integrands of (3.36) and (3.37), to obtain

$$
\partial_{-}\left[\left(\frac{1}{\pi \hbar} C_{i j}\left(\frac{1}{2} \partial_{+} \phi^{i} \partial_{+} \phi^{j}-\gamma^{i} \partial_{+} \partial_{+} \phi^{j}\right)\right)\right]=0 .
$$

Hence, we obtain the Noether current of the form

$$
\Theta=\frac{1}{2 \pi \hbar} C_{i j} \partial_{+} \phi^{i} \partial_{+} \phi^{j}-\frac{1}{\pi \hbar} C_{i j} \gamma^{i} \partial_{+} \partial_{+} \phi^{j},
$$

that obeys the current conservation equation $\partial_{-} \Theta=0$, whereby it must be true that $\Theta=\Theta\left(z, x^{+}\right)$. The expression (3.39) is the $3 \mathrm{~d}$ analogue of a component of the improved energy-momentum tensor in $2 \mathrm{~d}$ Toda theory.

Next, we wish to use the Noether current $\Theta\left(z, x^{+}\right)$to compute a $3 \mathrm{~d}$ analogue of a $\mathrm{W}$-algebra. We shall do so via an equivalent theory, namely a gauged version of the $3 \mathrm{~d}$ WZW model.

Equivalence between constrained and gauged 3d WZW models. We shall show that the constrained 3d WZW models, derived in section 3.1, can also be described by a gauged 3d WZW model which has the action

$$
\begin{aligned}
S_{3 \mathrm{~d} \text { GWZW }}\left[\widetilde{g}, \mathfrak{A}_{+}, \mathfrak{A}_{-}\right]= & S_{3 \mathrm{~d} \mathrm{WZW}}[\widetilde{g}]+\frac{1}{2 \pi \hbar} \int_{S^{1} \times \Sigma} d z \wedge d x^{+} \wedge d x^{-} \operatorname{Tr}\left(\mathfrak{A}_{+}\left(\partial_{-} \widetilde{g}\right) \widetilde{g}^{-1}+\left(\widetilde{g}^{-1} \partial_{+} \widetilde{g}\right) \mathfrak{A}_{-}\right. \\
& \left.+\mathfrak{A}_{+} \widetilde{g} \mathfrak{A}_{-} \widetilde{g}^{-1}-\mathfrak{A}_{-} \mu+\mathfrak{A}_{+} \nu\right)
\end{aligned}
$$

where the auxiliary gauge connections are defined by $\mathfrak{A}_{+}=h^{-1} \partial_{+} h, \mathfrak{A}_{-}=\widehat{h}^{-1} \partial_{-} \widehat{h}$ and $h, \widehat{h} \in H \subset \mathrm{SL}(N, \mathbb{C})$, while $\mu=\sum_{i} \mu_{i} R_{i}^{+}$and $\nu=\sum_{i} \nu_{i} R_{i}^{-}$. Recall that $R_{i}^{ \pm}$denote simple roots of $\mathfrak{s l}(N, \mathbb{C})$.

The action (3.40) can be shown to be invariant under the gauge transformations

$$
\begin{aligned}
\widetilde{g} & \rightarrow \alpha \widetilde{g} \beta^{-1}, \quad \alpha \in N^{-}, \beta \in N^{+} \\
h & \rightarrow h \alpha^{-1} \\
\widehat{h} & \rightarrow \widehat{h} \beta^{-1}
\end{aligned}
$$


where $N^{ \pm}$are the subspaces of $\operatorname{SL}(N, \mathbb{C})$ that are generated by ladder operators associated with \pm roots. Next, we take a variation of (3.40) with respect to the fields $\mathfrak{A}_{-}, \mathfrak{A}_{+}$and $\widetilde{g}$ which leads to the equations of motion

$$
\begin{aligned}
\widetilde{g}^{-1} \partial_{+} \widetilde{g}+\widetilde{g}^{-1} \mathfrak{A}_{+} \widetilde{g} & =\mu \\
-\partial_{-} \widetilde{g} \widetilde{g}^{-1}-\widetilde{g} \mathfrak{A}_{-} \widetilde{g}^{-1} & =\nu \\
-2 \partial_{-}\left(\widetilde{g}^{-1} \partial_{+} \widetilde{g}\right)-\widetilde{g}^{-1} \partial_{-} \mathfrak{A}_{+} \widetilde{g}+\widetilde{g}^{-1} \partial_{-} \widetilde{g} \widetilde{g}^{-1} \mathfrak{A}_{+} \widetilde{g}-\widetilde{g}^{-1} \mathfrak{A}_{+} \partial_{-} \widetilde{g}-\widetilde{g}^{-1} \partial_{+} \widetilde{g} \mathfrak{A}_{-} & \\
+\mathfrak{A}_{-} \widetilde{g}^{-1} \partial_{+} \widetilde{g}-\partial_{+} \mathfrak{A}_{-}+\mathfrak{A}_{-} \widetilde{g}^{-1} \mathfrak{A}_{+} \widetilde{g}-\widetilde{g}^{-1} \mathfrak{A}_{+} \widetilde{g} \mathfrak{A}_{-} & =0,
\end{aligned}
$$

respectively.

Finally, picking the partial gauge-fixing condition as $\mathfrak{A}_{+}=0=\mathfrak{A}_{-},{ }^{4}$ the equations of motion (3.42) become

$$
\begin{aligned}
\widetilde{g}^{-1} \partial_{+} \widetilde{g} & =\mu \\
-\partial_{-} \widetilde{g} \widetilde{g}^{-1} & =\nu \\
-2 \partial_{-}\left(\widetilde{g}^{-1} \partial_{+} \widetilde{g}\right) & =0,
\end{aligned}
$$

respectively. In particular, (3.43a) and (3.43b) are the current constraints (3.21) and (3.22). Furthermore, $(3.43 \mathrm{c})$ is the same equation of motion of the ungauged $3 \mathrm{~d}$ WZW model (see (3.9)). Following the steps seen in section 3.2, we can then obtain the $3 \mathrm{~d}$ Toda theory.

Therefore, the gauged 3d WZW model proposed here produces the same physics as the $3 \mathrm{~d}$ Toda theory. We shall now attempt to obtain $3 \mathrm{~d}$ analogues of $\mathrm{W}$-algebras from this equivalent description of the $3 \mathrm{~d}$ Toda theory, starting with a $3 \mathrm{~d}$ analogue of the Virasoro algebra.

Current algebra of gauged $\mathrm{SL}(2, \mathbb{C}) 3 \mathrm{~d}$ WZW model. For clarity, we shall first focus on the simplest case of the gauged $\mathrm{SL}(2, \mathbb{C}) 3 \mathrm{~d}$ WZW model that is equivalent to a $3 \mathrm{~d}$ analogue of Liouville theory. In this case, $\left[C_{i j}\right]=2$ and $\gamma_{i}=\frac{1}{2}$. We shall compute Poisson brackets in the $3 \mathrm{~d}$ gauged WZW model, where we shall take $x^{-}$to be the temporal direction. We shall also write $x^{+}=\xi$ for presentation purposes.

From (A.2), the symplectic form on the phase space is found to be

$$
\omega=\frac{1}{2} \int d \xi d z \operatorname{Tr}\left(\partial_{\xi}\left(\delta \widetilde{g}(z, \xi) \widetilde{g}^{-1}(z, \xi)\right) \wedge \delta \widetilde{g}(z, \xi) \widetilde{g}^{-1}(z, \xi)\right) .
$$

We moreover decompose the $3 \mathrm{~d}$ WZW current in terms of basis generators of $\mathrm{SL}(2, \mathbb{C})$, i.e.,

$$
J_{a}=-\frac{i}{2} \operatorname{Tr}\left(\tau_{a} \widetilde{g}^{-1}(z, \xi) \partial_{\xi} \widetilde{g}(z, \xi)\right),
$$

where $\tau_{a}$ denote $\operatorname{SL}(2, \mathbb{C})$ generators. Setting $\mathcal{O}=J_{a}\left(z^{\prime}, \xi^{\prime}\right)$ and $\zeta=\widetilde{g}(z, \xi)$ in (A.2), we obtain the Poisson bracket relations

$$
\left[J_{a}\left(z^{\prime}, \xi^{\prime}\right), \widetilde{g}(z, \xi)\right]_{\mathrm{PB}}=-\frac{i}{2} \widetilde{g}\left(z^{\prime}, \xi^{\prime}\right) \tau_{a} \delta\left(\xi-\xi^{\prime}\right) \delta\left(z-z^{\prime}\right) .
$$

\footnotetext{
${ }^{4}$ Note that the gauge-fixing condition $\mathfrak{A}_{+}=0=\mathfrak{A}_{-}$does not fix the gauge completely. There is a residual gauge symmetry $g \rightarrow \alpha g \beta^{-1}$, where $\alpha=\alpha\left(x^{+}\right)$and $\beta=\beta\left(x^{-}\right)$now depend only on the $x^{+}$and $x^{-}$directions, respectively.
} 
Likewise, setting $\mathcal{O}=J_{a}\left(z^{\prime}, \xi^{\prime}\right)$ and $\zeta=\widetilde{g}^{-1}(z, \xi)$ outputs

$$
\left[J_{a}\left(z^{\prime}, \xi^{\prime}\right), \widetilde{g}^{-1}(z, \xi)\right]_{\mathrm{PB}}=\frac{i}{2} \tau_{a} \widetilde{g}^{-1}\left(z^{\prime}, \xi^{\prime}\right) \delta\left(\xi-\xi^{\prime}\right) \delta\left(z-z^{\prime}\right) .
$$

Next, using (3.46) and (3.47), the Poisson bracket relations for $J_{a}$ are obtained as

$$
\left[J_{a}\left(z^{\prime}, \xi^{\prime}\right), J_{b}(z, \xi)\right]_{\mathrm{PB}}=\left(i \epsilon_{a b c} J^{c}(z, \xi) \delta\left(\xi-\xi^{\prime}\right)+\frac{1}{2} \delta_{a b} \delta^{\prime}\left(\xi-\xi^{\prime}\right)\right) \delta\left(z-z^{\prime}\right),
$$

where $\delta^{\prime}\left(\xi-\xi^{\prime}\right)=\partial_{\xi} \delta\left(\xi-\xi^{\prime}\right)$. This takes the form of the "analytically-continued" toroidal Lie algebra seen in [13].

Virasoro algebra of $\mathbf{3 d}$ Liouville theory. The next step is to derive the Poisson bracket relations for the $3 \mathrm{~d}$ analogue of the chiral Sugawara energy-momentum tensor,

$$
T(z, \xi)=J_{a}(z, \xi) J^{a}(z, \xi)
$$

Via (3.48), the Poisson bracket relations for $T(z, \xi)$ are obtained as

$$
\begin{aligned}
{\left[T\left(z^{\prime}, \xi^{\prime}\right), J_{a}(z, \xi)\right]_{\mathrm{PB}} } & =\left(\partial_{\xi} J_{a}(z, \xi) \delta\left(\xi-\xi^{\prime}\right)+J_{a}(z, \xi) \delta^{\prime}\left(\xi-\xi^{\prime}\right)\right) \delta\left(z-z^{\prime}\right) \\
{\left[T\left(z^{\prime}, \xi^{\prime}\right), T(z, \xi)\right]_{\mathrm{PB}} } & =\left(\partial_{\xi} T(z, \xi) \delta\left(\xi-\xi^{\prime}\right)+2 T(z, \xi) \delta^{\prime}\left(\xi-\xi^{\prime}\right)\right) \delta\left(z-z^{\prime}\right) .
\end{aligned}
$$

Next, we consider the Noether current given in (3.39). Via the Gauss decomposition (see (3.23)), and using the $3 \mathrm{~d}$ Toda equations of motion (3.29), we obtain the relation

$$
J_{3}=-\frac{1}{2} \partial_{\xi} \phi
$$

Thus, upon a rescaling, and using (3.52), we can also define (3.39) as

$$
\Theta(z, \xi)=T(z, \xi)-\partial_{\xi} J_{3}(z, \xi) .
$$

Finally, via (3.50) and (3.51), we obtain

$$
\left[\Theta\left(z^{\prime}, \xi^{\prime}\right), \Theta(z, \xi)\right]_{\mathrm{PB}}=\left(\partial_{\xi} \Theta(z, \xi) \delta\left(\xi-\xi^{\prime}\right)+2 \Theta(z, \xi) \delta^{\prime}\left(\xi-\xi^{\prime}\right)-\frac{1}{2} \delta^{\prime \prime \prime}\left(\xi-\xi^{\prime}\right)\right) \delta\left(z-z^{\prime}\right),
$$

which is a $3 \mathrm{~d}$ analogue of the classical Virasoro algebra derived from the $3 \mathrm{~d}$ Liouville theory.

In [14], where the analogue of (3.54) is derived for the $2 \mathrm{~d}$ Liouville theory, Moyal quantization is shown to lead to its central extension, whereby one arrives at the well-known form of the standard Virasoro algebra, that can be equivalently obtained via canonical quantization. We thus expect to obtain an analogous central extension to (3.54) via Moyal quantization, leading to an analogue of the standard, centrally extended Virasoro algebra. By analogy wth the $2 \mathrm{~d}$ case, this algebra ought to take the form

$$
\begin{aligned}
{\left[\widehat{\Theta}\left(z^{\prime}, \xi^{\prime}\right), \widehat{\Theta}(z, \xi)\right]=} & \left(\partial_{\xi} \widehat{\Theta}(z, \xi) \delta\left(\xi-\xi^{\prime}\right)+2 \widehat{\Theta}(z, \xi) \delta^{\prime}\left(\xi-\xi^{\prime}\right)\right. \\
& \left.+\left(k-\frac{1}{2} \eta^{2}\right) \delta^{\prime \prime \prime}\left(\xi-\xi^{\prime}\right)+k \delta^{\prime}\left(\xi-\xi^{\prime}\right)\right) \delta\left(z-z^{\prime}\right)
\end{aligned}
$$


where $k \in \mathbb{R}$ is a constant to be determined, and $\widehat{\Theta}(z, \xi)=T(z, \xi)-\eta \partial_{\xi} J_{3}(z, \xi)$, where $\eta$ is a deformation parameter.

When we replace $\operatorname{SL}(2, \mathbb{C})$ with $\operatorname{SL}(N, \mathbb{C})$, we also expect to obtain a Virasoro algebra of the form (3.55). Moreover, we should be able to derive analogous results for higher spin currents in the $3 \mathrm{~d}$ Toda theory via a generalized Sugawara construction, similar to how $\mathrm{W}$-algebra currents are constructed in $2 \mathrm{~d}$ Toda theory [15]. In doing so, we ought to obtain analogues of $\mathrm{W}$-algebras, similar in form to those that arise in $2 \mathrm{~d}$ Toda theory, but with generators having holomorphic dependence on the Riemann surface, $\Sigma$, and a delta function $\delta\left(z-z^{\prime}\right)$ appearing as an overall factor on the r.h.s. .

Note that, so far, we have taken $x^{-}$to be the temporal direction, and argued that this leads to a $3 \mathrm{~d}$ analogue of a $\mathrm{W}$-algebra via Moyal quantization. We could have equivalently taken $x^{+}$to be the temporal direction, and doing so should lead to another copy of the $\mathrm{W}$-algebra which is $x^{-}$-dependent instead.

\section{2d twisted sigma model from partially twisted 5d MSYM}

\subsection{2d A-model on Bogomolny moduli space as an effective theory}

Recall that we have started with the $5 \mathrm{~d}$ "GL"-twisted MSYM on $\mathcal{M}=I \times \mathbb{R}_{+} \times S^{1} \times \Sigma$. We would now like to derive a $2 \mathrm{~d}$ sigma model from our original setup, by taking the topological directions along $I \times \mathbb{R}_{+}$to be very large. To this end, we shall carry out the appropriate topological deformation of the metric, similar to the approach taken in [16].

Topological deformation. More explicitly, we shall consider a topological deformation of the form

$$
\begin{aligned}
d s^{2} & =\left(d x^{1}\right)^{2}+\left(d x^{2}\right)^{2}+\left(d x^{3}\right)^{2}+\left(d x^{4}\right)^{2}+\left(d x^{5}\right)^{2} \\
& \rightarrow \varepsilon^{-1}\left(\left(d x^{1}\right)^{2}+\left(d x^{2}\right)^{2}\right)+\left(d x^{3}\right)^{2}+\left(d x^{4}\right)^{2}+\left(d x^{5}\right)^{2},
\end{aligned}
$$

where $\varepsilon$ is taken to be very small. This deformation can be interpreted as making the volume of $I \times \mathbb{R}_{+}$large, relative to the volume of $S^{1} \times \Sigma$, which is equivalent to making the volume of $S^{1} \times \Sigma$ infinitesimally small. This results in an effective $2 \mathrm{~d}$ theory on $I \times \mathbb{R}_{+}$.

Let us now examine the $A$ - and $\varphi$ - dependent part of the 5 d action (2.1), which takes the form

$$
S_{5 \mathrm{~d} \mathrm{GL}}^{(A, \varphi)}=-\frac{1}{g_{5}^{2}} \int_{\mathcal{M}} d^{5} x \operatorname{Tr}\left(\frac{1}{2} \mathcal{F}_{\alpha \beta} \overline{\mathcal{F}}^{\alpha \beta}+4 \mathcal{F}_{\alpha \bar{z}} \overline{\mathcal{F}}^{\alpha}{ }_{z}+\left(D_{\alpha} \varphi^{\alpha}-2 i F_{z \bar{z}}\right)^{2}\right),
$$

where $z, \bar{z} \in \Sigma$ and $\alpha, \beta=1,2,3$, and $\mathcal{F}$ is the field strength corresponding to complex gauge fields $\mathcal{A}_{\alpha}=A_{\alpha}+i \varphi_{\alpha}$, where we recall that $\mathcal{A}_{z}=A_{z}$ and $\mathcal{A}_{\bar{z}}=A_{\bar{z}}$.

Following [16], we can decompose the fields as $A=A_{I \times \mathbb{R}_{+}}+A_{S^{1}}+A_{\Sigma}$ and $\varphi=$ $\varphi_{I \times \mathbb{R}_{+}}+\varphi_{S^{1}}$, and rewrite (4.2) as

$$
\begin{aligned}
S_{5 \mathrm{~d} G \mathrm{GL}}^{(A, \varphi)}= & -\frac{1}{g_{5}^{2}} \int_{\mathcal{M}} d^{5} x \operatorname{Tr}\left(\frac{1}{2} \mathcal{F}_{\widetilde{\alpha} \widetilde{\beta}} \overline{\mathcal{F}}^{\widetilde{\alpha} \widetilde{\beta}}+\mathcal{F}_{\tau \widetilde{\alpha}} \overline{\mathcal{F}}^{\tau \widetilde{\alpha}}+4 \mathcal{F}_{\tau \bar{z}} \overline{\mathcal{F}}^{\tau}{ }_{z}+4 \mathcal{F}_{\widetilde{\alpha} \bar{z}} \overline{\mathcal{F}}^{\widetilde{\alpha}}{ }_{z}\right. \\
& \left.+\left(D_{\widetilde{\alpha}} \varphi^{\widetilde{\alpha}}\right)^{2}+2 D_{\widetilde{\alpha}} \varphi^{\widetilde{\alpha}}\left(D_{\tau} \varphi^{\tau}-2 i F_{z \bar{z}}\right)+\left(D_{\tau} \varphi^{\tau}-2 i F_{z \bar{z}}\right)^{2}\right)
\end{aligned}
$$


where $x^{3}=\tau$, and $\widetilde{\alpha}, \widetilde{\beta}=1,2$. Next, we carry out the deformation (4.1), so that (4.3) becomes

$$
\begin{aligned}
S_{5 \mathrm{~d} G \mathrm{GL}}^{(A, \varphi)}= & -\frac{1}{g_{5}{ }^{2}} \int_{\mathcal{M}} d^{5} x \operatorname{Tr}\left(\frac{1}{2} \varepsilon \mathcal{F}_{\widetilde{\alpha} \widetilde{\beta}} \overline{\mathcal{F}}^{\widetilde{\alpha \beta}}+\mathcal{F}_{\tau \widetilde{\alpha}} \overline{\mathcal{F}}^{\tau \widetilde{\alpha}}+4 \varepsilon^{-1} \mathcal{F}_{\tau \bar{z}} \overline{\mathcal{F}}_{z}^{\tau}+4 \mathcal{F}_{\widetilde{\alpha} \bar{z}} \overline{\mathcal{F}}_{z}^{\widetilde{\alpha}}\right. \\
& \left.+\varepsilon\left(D_{\widetilde{\alpha}} \varphi^{\widetilde{\alpha}}\right)^{2}+2 D_{\widetilde{\alpha}} \varphi^{\widetilde{\alpha}}\left(D_{\tau} \varphi^{\tau}-2 i F_{z \bar{z}}\right)+\varepsilon^{-1}\left(D_{\tau} \varphi^{\tau}-2 i F_{z \bar{z}}\right)^{2}\right) .
\end{aligned}
$$

When we take the limit $\varepsilon \rightarrow 0$, terms proportional to $\varepsilon^{-1}$ will diverge. Hence, to ensure the finiteness of the action, it is necessary to impose the conditions

$$
\begin{aligned}
F_{\tau \bar{z}}-i D_{\bar{z}} \varphi_{\tau} & =0 \\
D_{\tau} \varphi^{\tau}-2 i F_{z \bar{z}} & =0
\end{aligned}
$$

Interestingly, (4.5) are the Bogomolny equations, otherwise known as 3d monopole equations. Hence, solutions of (4.5) span the moduli space of Bogomolny monopoles, which we shall denote as $\mathcal{M}_{\mathrm{B}}\left(G, S^{1} \times \Sigma\right)$.

2d sigma model action from "GL"-twisted 5d MSYM. Upon taking $\varepsilon \rightarrow 0$, and imposing the conditions (4.5), the remaining action becomes

$$
S_{5 \mathrm{~d} G \mathrm{GL}}^{(A, \varphi)}=-\frac{1}{g_{5}^{2}} \int_{\mathcal{M}} d^{5} x \operatorname{Tr}\left(\mathcal{F}_{p \widetilde{\alpha}} \overline{\mathcal{F}}^{p \widetilde{\alpha}}\right)
$$

where $p=3,4,5$.

In order to specify a solution of the Bogomolny equations, $A_{p}$ and $\varphi_{\tau}$, on $\mathcal{M}$, we shall specify a map $X: I \times \mathbb{R}_{+} \rightarrow \mathcal{M}_{\mathrm{B}}\left(G, S^{1} \times \Sigma\right)$. Then, we can write

$$
\begin{aligned}
& A_{p}\left(x^{1}, x^{2}, x^{3}, x^{4}, x^{5}\right)=A_{p}\left(x^{3}, x^{4}, x^{5} \mid X\left(x^{1}, x^{2}\right)\right) \\
& \varphi_{\tau}\left(x^{1}, x^{2}, x^{3}, x^{4}, x^{5}\right)=\varphi_{\tau}\left(x^{3}, x^{4}, x^{5} \mid X\left(x^{1}, x^{2}\right)\right) .
\end{aligned}
$$

We can express the variations of the solutions of the Bogomolny equations, $\delta A_{p}$ and $\delta \varphi_{\tau}$, in terms of cotangent vectors on $\mathcal{M}_{\mathrm{B}}\left(G, S^{1} \times \Sigma\right)$, up to gauge transformations, i.e.,

$$
\begin{aligned}
& \frac{\partial A_{p}}{\partial X^{I}}=\delta_{I} A_{p}+D_{p} E_{I} \\
& \frac{\partial \varphi_{\tau}}{\partial X^{I}}=\delta_{I} \varphi_{\tau}+\left[\varphi_{\tau}, E_{I}\right]
\end{aligned}
$$

where $I=1, \cdots, \operatorname{dim}\left(\mathcal{M}_{\mathrm{B}}\left(G, S^{1} \times \Sigma\right)\right)$, and where $E$ is identified with a gauge connection on $\mathcal{M}_{\mathrm{B}}\left(G, S^{1} \times \Sigma\right)$.

Next, we choose the gauge-fixing conditions $\overline{\mathcal{D}}^{\tau}\left(\delta_{I} A_{\tau}+i \delta_{I} \varphi_{\tau}\right)=0$ and $D_{z} \delta_{I} A_{\bar{z}}=0$. Noting that $\mathcal{A}_{\tilde{\alpha}}$ becomes auxiliary in the $\varepsilon \rightarrow 0$ limit, we integrate it out by setting

$$
\mathcal{A}_{\widetilde{\alpha}}=E_{I} \partial_{\widetilde{\alpha}} X^{I}
$$

assuming that the operator $\overline{\mathcal{D}}_{\tau} \mathcal{D}^{\tau}+4 D_{z} D_{\bar{z}}$ is invertible (this can be achieved via the inclusion of non-local operators on $\left.I \times \mathbb{R}_{+}\right)$where $\mathcal{D}_{\tau}=D_{\tau}+i\left[\varphi_{\tau}, \cdot\right]$. Hence, the action (4.6) 
becomes

$$
\begin{aligned}
S_{5 \mathrm{~d} G L}^{\prime(A, \varphi)} & =-\frac{1}{g_{5}{ }^{2}} \int_{I \times \mathbb{R}_{+}} d^{2} x\left(\int_{S^{1} \times \Sigma} d^{3} x \operatorname{Tr}\left(\delta_{I} A^{p} \delta_{J} A_{p}+\delta_{I} \varphi^{\tau} \delta_{J} \varphi_{\tau}\right)\right) \partial^{\widetilde{\alpha}} X^{I} \partial_{\alpha} X^{J} \\
& =\int_{I \times \mathbb{R}_{+}} d^{2} x G_{I J}^{\mathrm{B}} \partial^{\widetilde{\alpha}} X^{I} \partial_{\widetilde{\alpha}} X^{J}
\end{aligned}
$$

where we have identified the metric on $\mathcal{M}_{\mathrm{B}}\left(G, S^{1} \times \Sigma\right)$ as

$$
G^{\mathrm{B}}=-\frac{1}{g_{5}^{2}} \int_{S^{1} \times \Sigma} d^{3} x \operatorname{Tr}\left(\delta A^{p} \otimes \delta A_{p}+\delta \varphi^{\tau} \otimes \delta \varphi_{\tau}\right) .
$$

The action (4.10) is indeed the action of a bosonic sigma model governing maps $X$ : $I \times \mathbb{R}_{+} \rightarrow \mathcal{M}_{\mathrm{B}}\left(G, S^{1} \times \Sigma\right)$. The full, partially twisted 5d gauge theory should likewise reduce in the same way to a topological sigma model on $I \times \mathbb{R}_{+}$. This sigma model is an A-model in a particular symplectic structure on $\mathcal{M}_{\mathrm{B}}\left(G, S^{1} \times \Sigma\right)$. To see this, note that the non- $\mathcal{Q}$-exact term of the $5 \mathrm{~d}$ gauge theory includes a term proportional to

$$
\int_{I \times \mathbb{R}_{+} \times S^{1} \times \Sigma} d^{5} x \operatorname{Tr} \frac{1}{2} \varepsilon^{\alpha \beta \gamma}\left(\frac{1}{2} F_{\alpha 5} F_{\beta \gamma}-\partial_{\alpha}\left(F_{\beta 4} \phi_{\gamma}\right)\right) .
$$

In the reduction procedure we have outlined, this term reduces to an integral over a pullback to $I \times \mathbb{R}_{+}$of a symplectic form on $\mathcal{M}_{\mathrm{B}}\left(G, S^{1} \times \Sigma\right)$, that can be denoted as $\omega=\widetilde{\Psi} \omega_{K}^{B}$, with

$$
\omega_{K}^{\mathrm{B}}=\frac{1}{2 \pi} \int_{S^{1} \times \Sigma} d^{3} x \operatorname{Tr}\left(\delta \varphi_{\tau} \wedge \delta A_{4}-\delta A_{5} \wedge \delta A_{\tau}\right)
$$

where the subscript $K$ denotes that this is analogous to the symplectic structure $\omega_{K}$ on Hitchin's moduli space as defined in [9], if we identify $A_{5}$ with $\varphi_{4}$ in the latter. ${ }^{5}$ This implies that the $2 \mathrm{~d}$ sigma model is an A-model with symplectic structure $\widetilde{\Psi} \omega_{K}^{\mathrm{B}}$. In fact, the entire non- $\mathcal{Q}$-exact sector in (2.8) reduces as

$$
\frac{\widetilde{\Psi}}{4 \pi} \int_{I \times \mathbb{R}_{+} \times S^{1} \times \Sigma} d z \wedge \operatorname{Tr}(\mathcal{F} \wedge \mathcal{F}) \rightarrow \int_{I \times \mathbb{R}_{+}} X^{*}(\omega-i B),
$$

where $B=-\widetilde{\Psi} \omega_{I}^{\mathrm{B}}$ is a $B$-field, with

$$
\omega_{I}^{\mathrm{B}}=-\frac{1}{2 \pi} \int_{S^{1} \times \Sigma} d^{3} x \operatorname{Tr}\left(\delta A_{\tau} \wedge \delta A_{4}-\delta \varphi_{\tau} \wedge \delta A_{5}\right),
$$

which is the analogue of the symplectic structure $\omega_{I}$ on Hitchin's moduli space.

Note that, upon including in the 5d "GL"-twisted theory non-local operators along $I \times \mathbb{R}_{+}$that we expect to correspond to local operators in $3 \mathrm{~d}$ Toda theory, the sigma model we obtain will include non-local operators on the worldsheet, while the target space will have dependence on the points on $S^{1} \times \Sigma$ where the operators are located.

\footnotetext{
${ }^{5}$ In fact, upon dimensional reduction along $S^{1} \in \Sigma$ (for $\Sigma=\mathbb{R} \times S^{1}$ or $T^{2}$ ), we obtain the well-studied case where the target space is Hitchin's moduli space [9].
} 


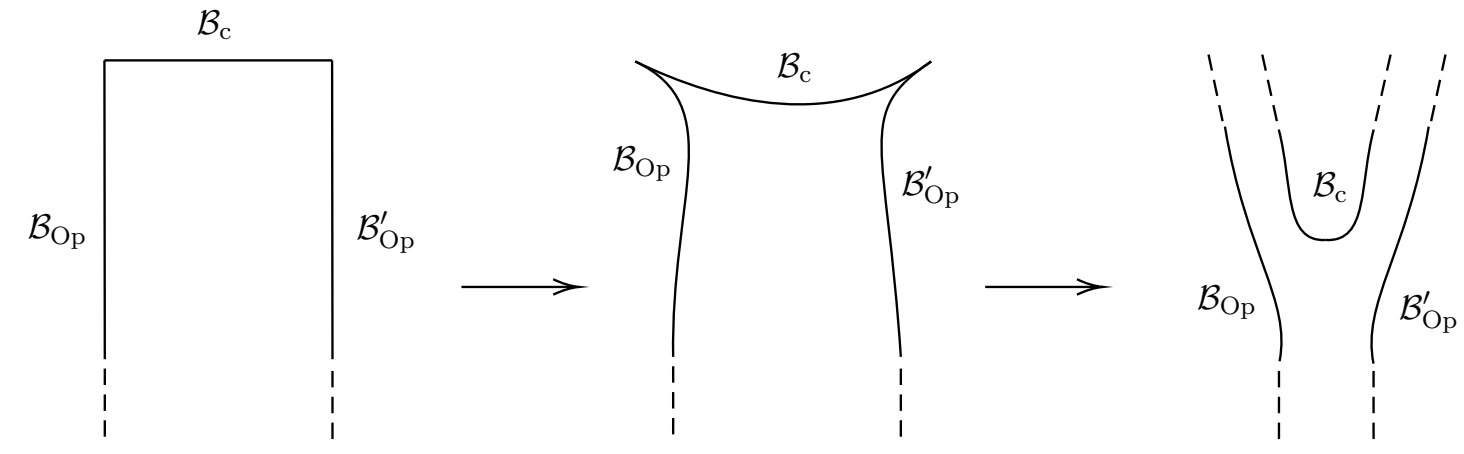

Figure 2. Topological deformation of sigma model worldsheet $I \times \mathbb{R}_{+}$.

\subsection{Physical states of 2d sigma model}

The NS5-type boundary condition of the $5 \mathrm{~d}$ gauge theory gives rise to a space-filling coisotropic A-brane, $\mathcal{B}_{\mathrm{c}}$, of the sigma model on $\mathcal{M}_{\mathrm{B}}\left(G, S^{1} \times \Sigma\right)$. Defining complex and symplectic structures analogous to those on Hitchin's moduli space (identifying $A_{5}$ with $\varphi_{4}$ as before), this is a $(B, A, A)$ brane. To see this, we first note that the NS5-type boundary condition implies Neumann boundary conditions on $\mathcal{A}_{\bar{z}}$ and $\mathcal{A}_{\tau}$, so the corresponding brane must be space-filling. Secondly, $\omega$ and $B$ satisfy $\left(\omega^{-1} B\right)^{2}=-1$, which implies that the brane is an A-brane in symplectic structure $\omega=\widetilde{\Psi} \omega_{K}^{\mathrm{B}}$. Finally, the $B$-field is of type $(1,1)$ in complex structure $I^{\mathrm{B}}$, implying that the brane is a B-brane in this complex structure.

On the other hand, the Nahm pole-type boundary conditions of the $5 \mathrm{~d}$ gauge theory give rise to Lagrangian branes $\mathcal{B}_{\mathrm{Op}}$ and $\mathcal{B}_{\text {Op }}^{\prime}$ in $\mathcal{M}_{\mathrm{B}}\left(G, S^{1} \times \Sigma\right)$. These are analogues of branes of opers in Hitchin's moduli space [3], and are both $(A, B, A)$ branes, since $\omega_{I}^{\mathrm{B}}$ and $\omega_{K}^{\mathrm{B}}$ both vanish on the support of these branes, and are maximal with this property.

It can be shown that the Lagrangian branes $\mathcal{B}_{\mathrm{Op}}$ and $\mathcal{B}_{\text {Op }}^{\prime}$ only intersect at one point, i.e., the origin. This implies that the space of states of the $\left(\mathcal{B}_{\mathrm{Op}}, \mathcal{B}_{\mathrm{Op}}^{\prime}\right)$ string contains only a single state. To find more states, we can topologically deform the worldsheet $I \times \mathbb{R}_{+}$ by pinching off the corners to infinity to form a Y-shaped worldsheet (see figure 2). This gives rise to $\left(\mathcal{B}_{\mathrm{c}}, \mathcal{B}_{\mathrm{Op}}\right)$ and $\left(\mathcal{B}_{\mathrm{c}}, \mathcal{B}_{\mathrm{Op}}^{\prime}\right)$ strings that originate from the corners of $I \times \mathbb{R}_{+}$. The physical space of states of each type of string corresponds to a space of $J^{\mathrm{B}}$-holomorphic sections of a bundle on the corresponding Lagrangian brane. Specifically, the two spaces of states can be computed (following [9]) to be

$$
\begin{aligned}
& \mathcal{H}_{\left(\mathcal{B}_{\mathrm{c}}, \mathcal{B}_{\mathrm{Op}}\right)}=H^{0}\left(\mathcal{B}_{\mathrm{Op}}, K_{\mathcal{B}_{\mathrm{Op}}}^{1 / 2}\right) \\
& \mathcal{H}_{\left(\mathcal{B}_{\mathrm{c}}, \mathcal{B}_{\mathrm{Op}}^{\prime}\right)}=H^{0}\left(\mathcal{B}_{\mathrm{Op}}^{\prime}, K_{\mathcal{B}_{\mathrm{OP}}^{\prime}}^{1 / 2}\right),
\end{aligned}
$$

where $K_{\mathcal{B}_{\mathrm{Op}}}$ is the canonical line bundle over the brane $\mathcal{B}_{\mathrm{Op}}$.

Moreover, the algebra of $\left(\mathcal{B}_{\mathrm{c}}, \mathcal{B}_{\mathrm{c}}\right)$ strings acts on the space of states of $\left(\mathcal{B}_{\mathrm{c}}, \mathcal{B}_{\mathrm{Op}}\right)$ and $\left(\mathcal{B}_{\mathrm{c}}, \mathcal{B}_{\mathrm{Op}}^{\prime}\right)$ strings, by attaching to the appropriate ends of the latter (see figure 3 ). This is just the quantized algebra of $J^{\mathrm{B}}$-holomorphic functions on $\mathcal{M}_{\mathrm{B}}\left(G, S^{1} \times \Sigma\right)$ [9], which are generated by $\mathcal{A}_{+}$and $\mathcal{A}_{-}$. The algebra of $\left(\mathcal{B}_{\text {Op }}, \mathcal{B}_{\text {Op }}\right)$ and $\left(\mathcal{B}_{\text {Op }}^{\prime}, \mathcal{B}_{\text {Op }}^{\prime}\right)$ strings likewise 


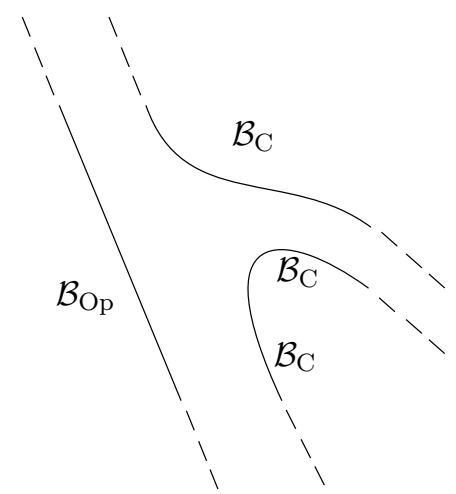

Figure 3. The action of the algebra of $\left(\mathcal{B}_{\mathrm{c}}, \mathcal{B}_{\mathrm{c}}\right)$ strings on the space of states of $\left(\mathcal{B}_{\mathrm{c}}, \mathcal{B}_{\mathrm{Op}}\right)$ can be realized by attaching the $\left(\mathcal{B}_{\mathrm{c}}, \mathcal{B}_{\mathrm{c}}\right)$ string to the end of the $\left(\mathcal{B}_{\mathrm{c}}, \mathcal{B}_{\mathrm{Op}}\right)$ string.

act on $\mathcal{H}_{\left(\mathcal{B}_{c}, \mathcal{B}_{\mathrm{OP}}\right)}$ and $\mathcal{H}_{\left(\mathcal{B}_{\mathrm{c}}, \mathcal{B}_{\mathrm{OP}}^{\prime}\right)}$ respectively. These are expected to be the same quantized algebra, albeit with different deformation parameters.

\section{The $3 \mathrm{~d}-2 \mathrm{~d}$ correspondence}

The 5d "GL"-twisted MSYM on $I \times \mathbb{R}_{+} \times S^{1} \times \Sigma$, with NS5-type boundary conditions on $\mathbb{R}_{+}$and Nahm pole-type boundary conditions on $I$, has given rise to two different effective descriptions.

In section 3, we localized "GL-twisted" 5d MSYM to the 4d CS theory at the origin of $\mathbb{R}_{+}$, which in turn, is equivalent to $3 \mathrm{~d}$ WZW models at the boundaries of $I$. Further implementing relevant constraints that originate from the Nahm pole-type boundary conditions (namely (2.13) and (2.16)), the 3d WZW models are then identified with 3d Toda theory on $S^{1} \times \Sigma$.

Via a gauged version of the $3 \mathrm{~d}$ WZW model, we also argued for the existence of $3 \mathrm{~d}$ analogues of $\mathrm{W}$-algebras, for which the corresponding modules must be identified with $3 \mathrm{~d}$ Toda states. There are two copies of such a W-algebra, corresponding to a choice of either $x^{+}$or $x^{-}$as the temporal direction.

In section 4, we argued that the $5 \mathrm{~d}$ "GL"-twisted theory gives rise, upon scaling up $I \times \mathbb{R}_{+}$, to a $2 \mathrm{~d}$ A-model of maps $X: I \times \mathbb{R}_{+} \rightarrow \mathcal{M}_{\mathrm{B}}\left(G, S^{1} \times \Sigma\right)$. Furthermore, via a topological deformation of the worldsheet $I \times \mathbb{R}_{+}$to a Y-shaped configuration, we also computed the physical states of the A-model.

Since the $\mathcal{Q}$-cohomology of states of the 5 d theory ought to remain invariant in reducing to these two effective descriptions, we may identify $3 \mathrm{~d}$ Toda states with physical states of the $2 \mathrm{~d}$ A-model. Hence, we establish a novel correspondence between the $3 \mathrm{~d}$ Toda theory and a topological sigma model with A-branes, i.e., we have

3d Toda theory

on

$$
S^{1} \times \Sigma
$$

Topological sigma model with A-branes

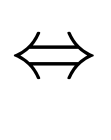

$$
\text { on }
$$$$
I \times \mathbb{R}_{+}
$$

with target $\mathcal{M}_{\mathrm{B}}\left(G, S^{1} \times \Sigma\right)$ 


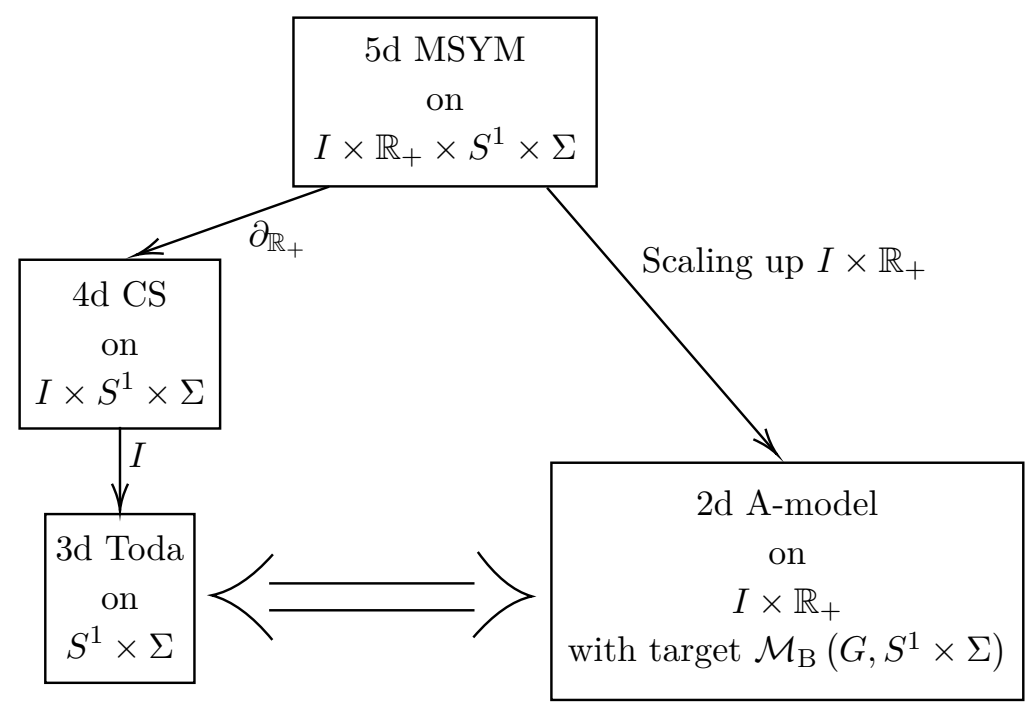

Figure 4. Outline of the steps taken in this paper, repeated here for brevity.

Mathematically, this implies that

$$
\begin{aligned}
& \text { Modules of } 3 \mathrm{~d} \mathrm{~W} \text {-algebras } \\
& \text { defined on } \\
& \Leftrightarrow \quad H^{0}\left(\mathcal{B}_{\mathrm{Op}}, K_{\mathcal{B}_{\mathrm{Op}}}^{1 / 2}\right) \otimes H^{0}\left(\mathcal{B}_{\mathrm{Op}}^{\prime}, K_{\mathcal{B}_{\mathrm{Op}}^{\prime}}^{1 / 2}\right) \\
& S^{1} \times \Sigma
\end{aligned}
$$

In other words,

Modules of 3d W-algebras defined on $S^{1} \times \Sigma$ are modules for the quantized algebra of $J^{\mathrm{B}}$-holomorphic functions on $\mathcal{M}_{\mathrm{B}}\left(G, S^{1} \times \Sigma\right)$.

The steps taken in arriving at these results are summarized in figure 4 .

A direct approach to understanding the $3 \mathrm{~d}-2 \mathrm{~d}$ correspondence described above is as follows. The $2 \mathrm{~d}$ A-model action is a sum of a $\mathcal{Q}$-exact term and a non- $\mathcal{Q}$-exact term that can be written as

$$
-\frac{\tilde{\Psi}}{4 \pi} X^{*}\left(\int_{S^{1} \times \Sigma} d z \wedge \delta \mathcal{A} \wedge \delta \mathcal{A}\right)=\tilde{\Psi} X^{*} \Omega_{J}^{B}
$$

where $\Omega_{J}^{B}$ is the analogue of the complex symplectic form $\Omega_{J}=-\frac{i}{4 \pi} \int_{C} \operatorname{Tr} \delta \mathcal{A} \wedge \delta \mathcal{A}$ on Hitchin's moduli space $\mathcal{M}_{H}(G, C)$, understood as the moduli space of flat $G_{\mathbb{C} \text {-connections }}$ on $C$. Since $\Omega_{J}$ is the natural symplectic form with respect to which $3 \mathrm{~d}$ analyticallycontinued Chern-Simons theory can be quantized via geometric quantization, one can similarly identify $\Omega_{J}^{B}$ as the relevant symplectic form for the quantization of $4 \mathrm{~d}$ Chern-Simons theory. Now, via the topological symmetry of the $2 \mathrm{~d}$ A-model, one can shrink the $I \times \mathbb{R}_{+}$ worldsheet to the half-line $\mathbb{R}_{+}$, thereby implementing the constraints coming from the Nahm pole-type boundary conditions into $\Omega_{J}^{B}$. The resulting symplectic form can then be identified with a symplectic form on the phase space of $3 \mathrm{~d}$ Toda theory, and in the $1 \mathrm{~d}$ description on $\mathbb{R}_{+}$reduces to a symplectic potential via Stokes' theorem. Localization of 
the quantum mechanical model means that the $\mathcal{Q}$-exact term drops out, and one is left with a path integral describing quantum mechanics on the phase space of $3 \mathrm{~d}$ Toda theory. Thus, the $3 \mathrm{~d}-2 \mathrm{~d}$ correspondence is essentially the identification of quantum $3 \mathrm{~d}$ Toda theory with the quantization of the phase space of classical $3 \mathrm{~d}$ Toda theory.

\section{Conclusion and future work}

In this work, we have derived a $3 \mathrm{~d}$ analogue of Toda theory, and shown that it is dual to a $2 \mathrm{~d}$ A-model on Bogomolny moduli space. Moreover, we have found that modules of $3 \mathrm{~d} \mathrm{~W}$ algebras are modules for a quantized algebra of holomorphic functions on the Bogomolny moduli space. The crucial ingredient is the fact that the $5 \mathrm{~d} \mathcal{N}=2 \mathrm{SYM}$ theory admits a partial twist analogous to the 4d GL-twist, that is topological-holomorphic.

This suggests a generalization. Namely, it will be interesting to define a GL-type partial twist (in two directions) for maximally supersymmetric Yang-Mills theory in 6d, whereby one expects to relate $4 \mathrm{~d}$ analogues of $\mathrm{W}$-algebras to a quantized algebra of certain holomorphic functions on the moduli space of instantons. Such 4d W-algebras should arise from a $4 \mathrm{~d}$ analogue of Toda theory, that should be the boundary dual of $5 \mathrm{~d}$ Chern-Simons theory with Nahm pole-type boundary conditions.

In addition, it would be interesting to embed the $5 \mathrm{~d}$ "GL"-twisted theory in a partial twist of the $6 \mathrm{~d} \mathcal{N}=(2,0)$ SCFT. This is likely to lead to a $3 \mathrm{~d}-3 \mathrm{~d}$ duality involving the $3 \mathrm{~d}$ Toda theory we have derived in this work.

We hope to explore these ideas in future work.

\section{Acknowledgments}

We would like to thank Sam Van Leuven, Gerben Oling, and Alessandro Tanzini for helpful correspondences. The results in this paper were presented at String Math 2020, and we would like to thank the audience, in particular, Nathan Seiberg, Cumrun Vafa, and Edward Witten for interesting comments and questions. This work is supported by the MOE Tier 2 grant R-144-000-396-112.

\section{A Poisson brackets}

Derivation of Poisson brackets from symplectic form. Generically, the Poisson brackets of two operators can be deduced from a non-degenerate symplectic form $[14,17]$ $\omega=\omega_{a b}(\zeta) \delta \zeta^{a} \wedge \delta \zeta^{b}$ via

$$
-\delta \mathcal{O}=\omega_{a b}(\zeta) \delta \zeta^{a}\left[\mathcal{O}, \zeta^{b}\right]_{\mathrm{PB}},
$$

where $\delta$ denotes a variation. Then, the Poisson bracket can be deduced by comparing the symplectic form with the variation of some operator $\mathcal{O}$.

In the continuous case, where $\omega=\int d x \omega(x) \delta \zeta(x) \wedge \delta \zeta(x)$, this becomes

$$
-\delta \mathcal{O}(x)=\int d y \omega(y) \delta \zeta(y)[\mathcal{O}(x), \zeta(y)]_{\mathrm{PB}} .
$$

In the same manner, the Poisson bracket can be deduced by comparing the symplectic form with the variation of some operator $\mathcal{O}(x)$. 
Open Access. This article is distributed under the terms of the Creative Commons Attribution License (CC-BY 4.0), which permits any use, distribution and reproduction in any medium, provided the original author(s) and source are credited.

\section{References}

[1] L.F. Alday, D. Gaiotto and Y. Tachikawa, Liouville Correlation Functions from Four-dimensional Gauge Theories, Lett. Math. Phys. 91 (2010) 167 [arXiv:0906.3219] [INSPIRE].

[2] N. Wyllard, $A_{N-1}$ conformal Toda field theory correlation functions from conformal $N=2$ $\mathrm{SU}(N)$ quiver gauge theories, JHEP 11 (2009) 002 [arXiv:0907.2189] [INSPIRE].

[3] N. Nekrasov and E. Witten, The Omega Deformation, Branes, Integrability, and Liouville Theory, JHEP 09 (2010) 092 [arXiv: 1002.0888] [INSPIRE].

[4] K. Costello, E. Witten and M. Yamazaki, Gauge Theory and Integrability, I, ICCM Not. 06 (2018) 46 [arXiv: 1709.09993] [INSPIRE].

[5] K. Costello, E. Witten and M. Yamazaki, Gauge Theory and Integrability, II, ICCM Not. 06 (2018) 120 [arXiv : 1802.01579] [InSPIRE].

[6] M. Ashwinkumar and M.-C. Tan, Unifying Lattice Models, Links and Quantum Geometric Langlands via Branes in String Theory, Adv. Theor. Math. Phys. 24 (2020) 1681 [arXiv: 1910.01134] [INSPIRE].

[7] B. Geyer and D. Mülsch, Higher dimensional analog of the Blau-Thompson model and $N_{T}=8, D=2$ Hodge type cohomological gauge theories, Nucl. Phys. B 662 (2003) 531 [hep-th/0211061] [INSPIRE].

[8] C. Cordova and D.L. Jafferis, Five-dimensional maximally supersymmetric Yang-Mills in supergravity backgrounds, JHEP 10 (2017) 003 [arXiv: 1305.2886] [INSPIRE].

[9] A. Kapustin and E. Witten, Electric-Magnetic Duality And The Geometric Langlands Program, Commun. Num. Theor. Phys. 1 (2007) 1 [hep-th/0604151] [InSPIRE].

[10] C. Elliott and V. Pestun, Multiplicative Hitchin Systems and Supersymmetric Gauge Theory, arXiv: 1812.05516 [INSPIRE].

[11] V. Mikhaylov, Teichmüller TQFT vs. Chern-Simons theory, JHEP 04 (2018) 085 [arXiv: 1710.04354] [INSPIRE].

[12] D. Gaiotto and E. Witten, Knot Invariants from Four-Dimensional Gauge Theory, Adv. Theor. Math. Phys. 16 (2012) 935 [arXiv:1106.4789] [INSPIRE].

[13] M. Ashwinkumar, Integrable Lattice Models and Holography, JHEP 02 (2021) 227 [arXiv: 2003. 08931] [INSPIRE].

[14] G. Jorjadze and G. Weigt, Poisson structure and Moyal quantization of the Liouville theory, Nucl. Phys. B 619 (2001) 232 [hep-th/0105306] [INSPIRE].

[15] P. Bouwknegt and K. Schoutens, W-symmetry in conformal field theory, Phys. Rept. 223 (1993) 183 [hep-th/9210010] [inSPIRE].

[16] M. Bershadsky, A. Johansen, V. Sadov and C. Vafa, Topological reduction of $4-D$ SYM to 2 - D sigma models, Nucl. Phys. B 448 (1995) 166 [hep-th/9501096] [InSPIRE].

[17] E. Witten, Non-Abelian bosonization in two-dimensions, Commun. Math. Phys. 92 (1984) 455 [INSPIRE]. 